\title{
Nicht invasive Diagnostik der koronaren Herzerkrankung: CT oder MRT?
}

Susan Rosemeier, Christopher Berger, Christian Krieghoff, Matthias Gutberlet

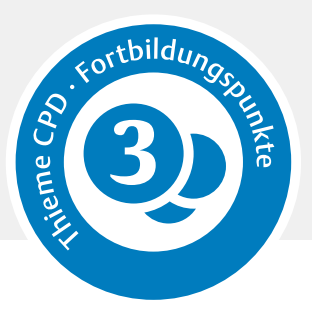

Eine zeitige Diagnose ist entscheidend, um fatale Ereignisse wie Herzinfarkt und plötzlichen Herztod verhindern zu können. Deshalb nehmen die kardiale CT und MRT in den Leitlinien als auch in der klinischen Realität einen immer größeren Stellenwert in der nicht invasiven Diagnostik KHK ein.

\section{Pathophysiologie der koronaren Herzkrankheit}

Die koronare Herzkrankheit (KHK) ist eine Ausprägungsform der Arteriosklerose, bei der es zu einer fortschreitenden Versteifung und Einengung der Herzkranzgefäße kommt. Grundsätzlich ist die Arteriosklerose jedoch eine „systemische“ Erkrankung, d. h. sie kann alle arteriellen Gefäße des Körpers erfassen [1].

Die Einengung der Herzkranzgefäße bei der KHK kann dazu führen, dass es zu einer nicht mehr ausreichenden Durchblutung des Herzmuskels kommt. Zunächst tritt diese Durchblutungsstörung in der Regel nur bei stärkerer körperlicher oder emotionaler Belastung auf, kann

FACHARZT- UND MTRA-FACHKRAFTWISSEN HERZ - 10. DEUTSCHE KARDIODIAGNOSTIK-TAGE 2018 MIT 11. LEIPZIGER SYMPOSIUM NICHTINVASIVE KARDIOVASKULÄRE BILDGEBUNG, 22. BIS 24. FEBRUAR 2018, KONGRESSHALLE AM ZOO, LEIPZIG

Die Zeit ist wie im Flug vergangen - 2018 feiern die Deutschen Kardiodiagnostik-Tage das 10-jährige Jubiläum vom 22. bis 24. Februar in Leipzig. Die kardiovaskuläre Bildgebung spielt im klinischen Alltag zur Diagnosestellung, vor Einleitung einer Therapie und Abschätzung der Prognose, eine zunehmend wichtige Rolle. Die nichtinvasive kardiale Bildgebung mit der Echokardiografie, dem kardialen MRT, der CT und auch der Nuklearmedizin liefert dazu immer bessere Grundlagen. Durch den rasanten technischen Fortschritt ergeben sich aber immer wieder neue Aspekte, die in den klinischen Alltag zusammen mit den aktualisierten Leitlinien einzuordnen aber bei hochgradigen Einengungen oder einem akuten Verschluss des Gefäßes - wie es bei einem Infarkt auftritt auch schon in Ruhe vorhanden sein.

Bei Belastung hat der Herzmuskel einen stärkeren Sauerstoffbedarf, der durch eine Steigerung der Herzmuskeldurchblutung gewährleistet werden muss. Bei der KHK liegt somit ein Missverhältnis zwischen Sauerstoff- bzw. Durchblutungsbedarf und Angebot vor, was auch als Koronarinsuffizienz bezeichnet wird. Das Ausmaß dieses Missverhältnisses ist unter anderem abhängig vom Ausmaß der Gefäßeinengung [2].

sind. Einsteiger wie auch Experten aus Radiologie, Kardiologie, Herzchirurgie, Innerer Medizin, Allgemeinmedizin und Nuklearmedizin finden eine Vielzahl von Zertifizierungsoptionen und Workshops sowie ein umfangreiches Programm speziell für MTRA/RT.

Nutzen Sie den Artikel des wissenschaftlichen Leiters der Veranstaltung - Professor Dr. med. Matthias Gutberlet, Universität Leipzig, Herzzentrum - zur Vorbereitung auf die 10. Deutschen Kardiodiagnostiktage und sammeln Sie schon jetzt CPD-Fortbildungspunkte. Das komplette Programm finden Sie unter www.kardiodiagnostik.de
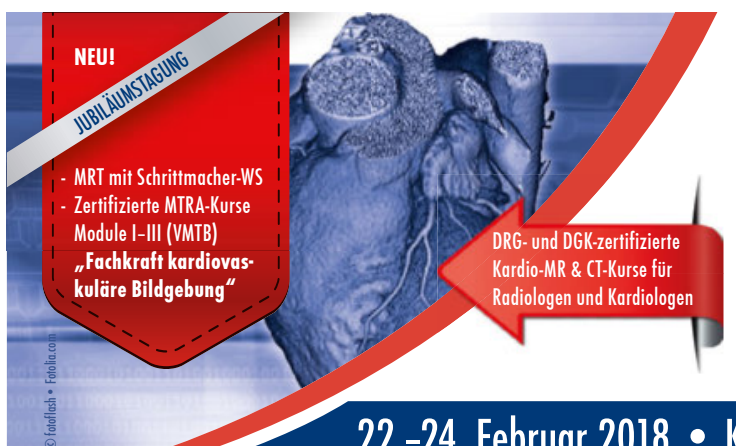

\section{Deutsche Kardiodiagnostik-Tage 2018}

mit 11. Leipziger Symposium Nichtinvasive Kardiovaskuläre Bildgebung Bildgebung zur Diagnostik, Prognose und Therapieplanung kardiovaskulärer Erkrankungen

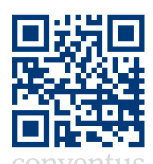


Merke

Die koronare Herzkrankheit (KHK) ist eine

Ausprägungsform der Arteriosklerose, bei der es zu einer fortschreitenden Versteifung und Einengung der Herzkranzgefäße kommt.

\section{Klinisches Erscheinungsbild der koronaren Herzkrankheit}

Üblicherweise kann die Ruhedurchblutung auch bei einer Gefäßeinengung von über 75 \% durch die eigenen Regulationsmechanismen des Herzens noch gewährleistet werden. Unter Belastung und einem höheren Sauerstoffund damit Durchblutungsbedarf reichen diese Mechanismen aber nicht mehr aus, und es kommt zu einer belastungsinduzierten Durchblutungsstörung - auch Ischämie genannt. Diese belastungsinduzierten, gelegentlich aber auch in Ruhe auftretenden Ischämien, führen zu typischen Beschwerden. Diese sind im Allgemeinen durch Belastung aber auch emotionalen Stress ausgelöste Brustschmerzen, die in den Hals oder den linken Arm ausstrahlen können. Man nennt sie deshalb auch Angina pectoris (wörtlich: Brustenge). Sie halten in der Regel nur wenige Minuten an und bessern sich schnell in Ruhe oder auf Gabe des Medikaments Nitroglycerin (meist als sublinguales Nitrospray), welches die Gefäße weit stellt. Die Beschwerden können sich aber auch nur in Form von belastungsinduzierter Atemnot, der sog. Dyspnoe, äußern, was die Differenzialdiagnose schwierig macht, oder auch ganz fehlen. Dies ist insbesondere bei der Risikogruppe der Diabetiker, aber auch bei Frauen häufiger der Fall.

Die KHK mit wiederkehrenden Durchblutungsstörungen kann neben dem Symptom einer Angina pectoris, zu einer begrenzten oder unwiderruflichen Funktionseinschränkung der Herzkammern bis hin zum Herzinfarkt führen, weshalb eine rechtzeitige Diagnose und Therapie vor einem möglichen unwiederbringlichen Zelluntergang wichtig ist.

\section{Merke}

Die KHK mit wiederkehrenden Durchblutungsstörungen kann neben dem Symptom einer Angina pectoris zu einer begrenzten oder unwiderruflichen Funktionseinschränkung der Herzkammern bis hin zum Herzinfarkt führen.

\section{Wann soll eine nicht invasive Diagnostik der koronaren Herzerkrankung durch- geführt werden?}

\section{Indikationen für eine nicht invasive KHK- Diagnostik - MRT oder CT?}

In Abhängigkeit von ihrer diagnostischen Genauigkeit, Verfügbarkeit, lokalen Erfahrung und der für den Patienten individuellen Kosten-Nutzen-Analyse haben verschiedene diagnostische Verfahren einen unterschiedlichen Stellenwert. Dies schlägt sich auch in den Leitlinien der verschiedenen Fachgesellschaften bis hin zur von der Bundesärztekammer (BÄK), Kassenärztlichen Bundesvereinigung (KBV) zusammen mit den Fachgesellschaften herausgegebenen, aktuellen Versorgungsleitlinie „Chronische KHK“ nieder [3],[4],[5]. Zur Beurteilung des individuellen Risikos auf das Vorliegen einer KHK werden gerne Scores zur Risikoabschätzung verwendet. Einer der am weitesten verbreiteten Scores für die Abschätzung des Risikos auf das Vorliegen einer KHK ist der Diamond-Forrester Score.

Der Diamond-Forrester Score ist für die invasive Koronardiagnostik entwickelt worden und gilt streng genommen nur für diese, kann aber auch zur groben Indikationsabschätzung für nicht invasive Tests genutzt werden. Hierbei muss allerdings dann berücksichtigt werden, dass das individuelle Risiko eines Patienten eventuell überschätzt wird [6],[7],[8]. Die Aussagekraft eines diagnostischen Tests hängt entscheidend von der zugrunde liegenden Vortestwahrscheinlichkeit ab und ist bei „mittlerer“ Vortestwahrscheinlichkeit in der Regel am besten, d. h., wenn die Wahrscheinlichkeit für das Vorliegen einer KHK zwischen 15-85\% liegt. Der Diamond-Forrester-Score bezieht nur 3 Faktoren zur Abschätzung der Vortestwahrscheinlichkeit heran und ist deshalb relativ einfach anwendbar ( $\vee$ Tab. 1.1).

- Tab. 1.1 Diamond-Forrester-Score: 3 Faktoren (Alter, Geschlecht, Beschwerdesymptomatik) zur Abschätzung der Vortestwahrscheinlichkeit (VTW) auf das Vorliegen einer Koronaren Herzerkrankung (KHK).

\begin{tabular}{|c|c|c|c|c|c|}
\hline Alter & Geschlecht & $\begin{array}{l}\text { Typische/eindeutige } \\
\text { Angina pectoris }\end{array}$ & $\begin{array}{l}\text { Atypische/wahrscheinliche } \\
\text { Angina pectoris }\end{array}$ & $\begin{array}{l}\text { Nicht anginöse } \\
\text { Brustschmerzen }\end{array}$ & Asymptomatisch \\
\hline$<39$ & $\begin{array}{l}\text { - Mann } \\
\text { - Frau }\end{array}$ & $\begin{array}{l}\text { - mittlere VTW } \\
\text { - mittlere VTW }\end{array}$ & $\begin{array}{l}\text { - mittlere VTW } \\
\text { - sehr niedrige VTW }\end{array}$ & $\begin{array}{l}\text { - } \text { niedrige VTW } \\
\text { - sehr niedrige VTW }\end{array}$ & $\begin{array}{l}\text { - sehr niedrige VTW } \\
\text { - sehr niedrige VTW }\end{array}$ \\
\hline $40-49$ & $\begin{array}{l}\text { - Mann } \\
\text { - Frau }\end{array}$ & $\begin{array}{l}\text { - } \text { hohe VTW } \\
\text { - mittlere VTW }\end{array}$ & $\begin{array}{l}\text { - mittlere VTW } \\
\text { - } \text { niedrige VTW }\end{array}$ & $\begin{array}{l}\text { - mittlere VTW } \\
\text { - sehr niedrige VTW }\end{array}$ & $\begin{array}{l}\text { - } \text { niedrige VTW } \\
\text { - sehr niedrige VTW }\end{array}$ \\
\hline $50-59$ & $\begin{array}{l}\text { - Mann } \\
\text { - Frau }\end{array}$ & $\begin{array}{l}\text { - } \text { hohe VTW } \\
\text { - mittlere VTW }\end{array}$ & $\begin{array}{l}\text { - mittlere VTW } \\
\text { - mittlere VTW }\end{array}$ & $\begin{array}{l}\text { - mittlere VTW } \\
\text { - sehr niedrige VTW }\end{array}$ & $\begin{array}{l}\text { - } \text { niedrige VTW } \\
\text { - sehr niedrige VTW }\end{array}$ \\
\hline$>60$ & $\begin{array}{l}\text { - Mann } \\
\text { - Frau }\end{array}$ & $\begin{array}{l}\text { - hohe VTW } \\
\text { - hohe VTW }\end{array}$ & $\begin{array}{l}\text { - mittlere VTW } \\
\text { - mittlere VTW }\end{array}$ & $\begin{array}{l}\text { - mittlere VTW } \\
\text { - mittlere VTW }\end{array}$ & $\begin{array}{l}\text { - } \text { niedrige VTW } \\
\text { - } \text { niedrige VTW }\end{array}$ \\
\hline
\end{tabular}


Das in Tab. 1.1 vorgestellte sehr einfache Schema ist dem kardialen MRCT-Registry der European Society of Cardiovascular Radiology (ESCR) entnommen [9]. Etwas detailliertere Schemata mit genauen Prozentangaben können der Literatur entnommen werden [4]. Es ist bekannt, dass insbesondere die Fahrradergometrie, also das sog. Belastungs-EKG, nur eine sehr niedrige Sensitivität für die Primärdiagnostik der KHK aufweist. Nicht invasive Ischämie, also die Durchblutungsstörung, nachweisende Methoden hingegen, wie

- die Stress-Echokardiografie

- die Stress-Myokardszintigrafie [10]

- die Stress-MRT

weisen eine hohe Sensitivität und Spezifität auf. Dies wurde in großen Studien ermittelt. Trotz einiger theoretischer und in großen Studien bestätigter Vorteile der Stress-MRT, wie z. B.

- eine hohe räumliche Auflösung

- eine fehlende Strahlenexposition

- keine Abhängigkeit von einem ausreichenden Schallfenster

im Vergleich zu den erstgenannten Methoden, werden alle 3 Methoden nach den aktuellen Leitlinien [3],[4],[5] als gleichwertig eingestuft und sollten in Abhängigkeit von der Verfügbarkeit und der lokalen Erfahrung der Anwender mit dieser Methode eingesetzt werden [4],[5]. Aus diesem Grunde werden sie in der aktuellen von der Bundeärztekammer (BÄK), der Kassenärztlichen Bundesvereinigung (KBV) und dem AWMF herausgegebenen Nationalen Versorgungsleitlinie - „Chronische KHK“ [5] für alle Patienten mit mittlerer Vortestwahrscheinlichkeit, also zwischen $15-85 \%$, empfohlen.

Für die Koronar-CT ergibt sich als einziges nicht invasives Verfahren, welches die Koronarmorphologie direkt darstellen kann, eine Besonderheit, weswegen sie vor allem für Patienten mit niedriger mittlerer Vortestwahrscheinlichkeit (15-50\%) empfohlen wird. Sie weist einen besonders hohen negativen prädiktiven Wert zwischen 98$100 \%$ auf, d. h., wenn die Koronar-CT eine stenosierende, relevante KHK ausschließt, dann hat der Patient auch keine relevante KHK. Bei der Beurteilung der hämodynamischen Relevanz einer in der CT nachgewiesenen Einengung, also der Frage, ob diese Einengung tatsächlich zu einer relevanten Durchblutungsstörung am Herzen des Patienten führt und damit behandelt werden muss, sind die Ergebnisse der CT weniger gut. Aus diesem Grund muss dann oft die Diagnostik noch um ein zusätzliches ischämienachweisendes Verfahren ergänzt oder die Patienten direkt dem invasiven Herzkatheter zugeführt werden. Aus diesem Grunde wird empfohlen, die Koronar-CT vor allem bei den Patienten mit niedriger mittlerer Vortestwahrscheinlichkeit (15-50\%) [4],[5] durchzuführen.

Merke

Patienten mit niedriger ( $<15 \%$ ) und hoher (> $85 \%$ )

Vortestwahrscheinlichkeit für das Vorliegen einer KHK haben bisher keine Indikation für eine Kardio-MRT oder Kardio-CT. Letztere haben eine Indikation für eine invasive Diagnostik.

\section{Kardiales MRCT-Registry der ESCR - Werden die Indikationen in Deutschland eingehalten?}

Nach den Ergebnissen des MRCT-Registry der ESCR, in der mit Stand Oktober 2017 ca. 180000 kardiale MRTs und CTs registriert wurden, werden die Untersuchungen in der Mehrzahl der Fälle auch entsprechend der aktuellen Leitlinien durchgeführt. Die Hauptindikation für eine kardiale CT stellt nach dem MRCT-Registry der ESCR der Verdacht auf eine stenosierende KHK dar. Diese Untersuchungsmethode liegt mit durchschnittlich $67 \%$ aller Untersuchungen deutlich vor der EKG-getriggerten Aortenbulbusdarstellung vor geplanter Transkatheter-Aortenklappenimplantation (Transcatheter Aortic Valve Implantation, TAVI). Bei den kardialen MRT-Untersuchungen liegt mit 38 \% die Evaluation von Kardiomyopathien/Myokarditis als Hauptindikationen vorne, dicht gefolgt von der KHK-Evaluation mit $33 \%$ aller CMR-Indikationen auf Platz 2. In der Mehrzahl der Fälle (MRT: 69 \%, CT: 68 \%) wurden entsprechend der Leitlinien [3],[4],[5] auch nur Patienten mit mittlerer bzw. intermediärer Vortestwahrscheinlichkeit eingeschlossen.

Die Ergebnisse des MRCT-Registry der ESCR sind besonders repräsentativ für die Situation in Deutschland, weil für die radiologischen Ärzte in Deutschland, die eine Zertifizierung in kardialer CT und/oder kardialer MRT der DRG (Q1-Q3-Level) erwerben wollen, eine Dokumentation ihrer selbst durchgeführten Fälle im Registry Voraussetzung ist. Ab Oktober 2017 können auch MTRA nach vorheriger Anmeldung ihrer Einrichtung auf der Webseite www.mrctregistry.org eine individuelle Anmeldung und Dokumentation ihrer selbst durchgeführten Fälle für die Erlangung der Qualifikation „Fachkraft für kardiovaskuläre Bildgebung“ der VMTB vornehmen. Danach können die entsprechenden Zertifikate für die Beantragung online erstellt werden ( $\triangleright$ Abb. 1).

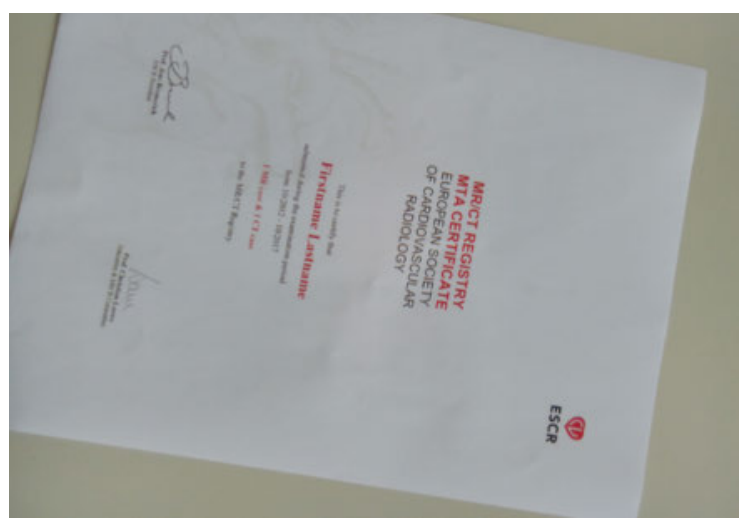

Abb. 1 Musterbeispiel eines MTRA-Zertifikats der ESCR für im MRCT-Registry dokumentierte kardiale MRT- und CT-Fälle. 


\section{Wie soll eine nicht invasive Diagnostik der koronaren Herzerkrankung durch- geführt werden?}

\section{Was ist bei der Durchführung einer kardialen CT bzw. MRT zu beachten?}

Die Ursache der KHK kann aufgrund der zugrunde liegenden Pathophysiologie entweder direkt durch Darstellung der morphologischen Veränderungen an den Herzkranzgefäßen oder indirekt durch den Nachweis der belastungsinduzierten Durchblutungsstörung erfolgen. Hieraus ergeben sich zwei elementare Unterschiede für die Durchführung der beiden Methoden Kardio-CT und Kardio-MRT.

Bei der Kardio-CT sollte die Herzfrequenz möglichst langsam sein, um gute Bilder zu erzeugen, bei der Kardio-MRT muss man den Patienten „belasten“ und damit medikamentös den Sauerstoffverbrauch bzw. die Durchblutung erhöhen. Dies führt entweder direkt oder indirekt zu einer Erhöhung der Herzfrequenz. Dementsprechend sind für beide Methoden unterschiedliche Vorbereitungen zu treffen und Kontraindikationen zu berücksichtigen.

\section{Koronar-CT}

Technische und personelle Grundvoraussetzungen

Die Durchführung einer Koronar-CT stellt hohe Ansprüche an Gerätetechnik und Fachpersonal. Eine gute Übersicht bieten die Leitlinien der Society of Cardiovascular Computed Tomography (SCCT) zur Durchführung der Koronar-CT [11].

- Verwendet werden sollten mindestens:

- ein 64-Zeilen-CT mit niedriger Rotationszeit

- eine EKG-Synchronisation

- eine hohe isotrope Detektorauflösung

- Eine Kontrastmittelpumpe (Powerinjektor) mit einem Doppelkopfsystem, um biphasische (Kontrastmittel, $\mathrm{NaCl}$ ) und/oder triphasische (Kontrastmittel,

Kontrastmittel $+\mathrm{NaCl}, \mathrm{NaCl}$ ) Kontrastmittelprotokolle anwenden zu können, sollte ebenfalls vorhanden sein.

- Für eine bestmögliche Kontrastierung der Koronararterien wird eine hohe Flussrate des Kontrastmittels (4-7 ml / s) empfohlen.

- Betablocker und Nitrolingual werden als Prämedikation für ein Koronar-CT verwendet.

- Für die schnelle Behandlung von Nebenwirkungen durch Kontrastmittel oder Prämedikation müssen Notfallmedikamente bereitstehen, wie z. B. für einen allergischen Kontrastmittelzwischenfall H1-, H2-Blocker sowie Kortikosteroide.

- Des Weiteren müssen MTRA und Ärzte im Umgang mit Notfallsituationen, Medikamenten und Defibrillatoren geschult sein, um im Ernstfall schnell und sicher handeln zu können; Grundvoraussetzung dafür ist die kontinuierliche Teilnahme an Rea-Kursen und entsprechenden Fortbildungen.

\section{CHECKLISTE}

Geräteanforderungen nach SCCT-Guidelines:

- $\geq 64$ Zeilen

- Rotationszeit $\leq 350 \mathrm{~ms}$

- EKG-Synchronisationstechniken

- retrospektives EKG-Gating mit Dosismodulation

- prospektive EKG-Triggerung

- Isotrope Auflösung < $1 \mathrm{~mm}$

\section{Vorbereitung des Patienten}

Die optimale Bildqualität ist insbesondere abhängig von der aktiven Mitarbeit des Patienten. Die umfassenden Informationen über

- Art der Untersuchung

- Ablauf und Anweisungen zum korrekten Verhalten vor der Untersuchung

tragen zu einer für den Patienten als ruhigere Atmosphäre wahrgenommene Gesamtsituation bei und helfen bei der Aufnahme qualitativ hochwertiger Bilder.

Optimalerweise sollten Patienten vor der Untersuchung keine koffeinhaltigen Produkte wie z. B. Kaffee oder Energydrinks zu sich genommen haben. Die Einnahme stellt zwar keine Kontraindikation dar, kann aber die für eine adäquate Bildqualität bzw. den Einsatz dosissparender Protokolle notwendige Herzfrequenzsenkung erschweren. Die patienteneigene Medikation sollte normal fortgesetzt werden. Eine Ausnahme hierbei stellt das Metformin dar, welches nur bei einer glomerulären Filtrationsrate von mindestens $45 \mathrm{ml} / \mathrm{min} / 1,73 \mathrm{~m}^{2}$ normal weiter genommen werden kann. Bei einer GFR von 30-44 ml/min/1,73 m² sollte Metformin jeweils $48 \mathrm{~h}$ vor und nach der Untersuchung pausiert und erst nach Kontrolle der Retentionsparameter wieder eingenommen werden. Bei einer GFR <30 $\mathrm{ml} / \mathrm{min} / 1,73 \mathrm{~m}^{2}$ sind sowohl Metformin- als auch eine Kontrastmittelgabe kontraindiziert.

Wie bei allen Untersuchungen mit jodhaltigen Kontrastmitteln sollten die gängigen Laborparameter Kreatinin (Krea) und die daraus berechnete glomeruläre Filtrationsrate (GFR) und das Thyreoidea-stimulierende Hormon (TSH) überprüft werden, um die normale Funktion von Niere (Kreatinin < $90 \mu \mathrm{mol} /$ l, GFR $>45 \mathrm{ml} / \mathrm{min}$ ) und Schilddrüse (TSH > 0,28 $\mu \mathrm{U} / \mathrm{ml}$ ) zu bestätigen. Des Weiteren sollte eine Allergieanamnese durchgeführt werden.

Bezüglich einer möglichen Prämedikation ( $\triangleright$ Tab. 1.2), typischerweise Nitrolingualspray und eventuell Betablocker, sollten Kontraindikationen ausgeschlossen werden ( Tab. 1.3).

Betablocker senken die Herzfrequenz. Für eine optimale Koronardarstellung sollte die Herzfrequenz - je nach Scannertyp - bei $\leq 60-70$ / min und der Rhythmus des 
- Tab. 1.2 Anwendung der Prämedikation.

\begin{tabular}{|c|c|c|}
\hline & Betablocker & Nitroglycerin \\
\hline Wirkmechanismus & $\begin{array}{l}\text { - selektive Hemmung von } \beta 1 \text {-Rezeptoren } \\
\text { - negative Chronotropie und somit Senkung der } \\
\text { Herzfrequenz und Herzfrequenzvariabilität }\end{array}$ & $\begin{array}{l}\text { direkte vasodilatatorische Effekte durch } \\
\text { Relaxation vaskulärer Muskelzellen der } \\
\text { Koronararterien und damit Verbesserung } \\
\text { der Beurteilbarkeit auch kleiner Gefäße }\end{array}$ \\
\hline Indikationen & $\begin{array}{l}\text { - regelmäßige Herzfrequenz < } 60 \text { bpm: } \\
\text { keine Betablockergabe zwingend nötig } \\
\text { - Herzfrequenz > } 60 \text { bpm oder Arrhythmie: } \\
\text { Betablockergabe indiziert }\end{array}$ & $\begin{array}{l}\text { bei fehlenden Kontraindikationen zur } \\
\text { Verbesserung der Beurteilbarkeit }\end{array}$ \\
\hline Dosierung & $\begin{array}{l}\text { - } 50 \text { mg Metoprolol per os } \\
\text { Gabe: } 12 \text { h vor der Untersuchung } \\
\text { - } 50-100 \text { mg Metoprolol per os } \\
\text { Gabe: } 1 \text { h vor Scan } \\
\text { - Alternativ bis } 5 \text { x } 5 \text { mg Metoprolol } \\
\text { i. v. unter ständiger Kontrolle bis zum Erreichen } \\
\text { der Zielfrequenz. } \\
\text { Gabe: direkt vor der Untersuchung }\end{array}$ & $\begin{array}{l}400-800 \mu \text { g sublingual } \\
(2 \text { Tabletten oder } 2 \text { Hübe Spray) }\end{array}$ \\
\hline Wirkeintritt & $\begin{array}{l}\text { - Per os: } 1,5-2 \mathrm{~h} \\
\text { - i. v.-Gabe: } 5-10 \mathrm{~min}\end{array}$ & $\begin{array}{l}\text { Maximum der Koronardilatation: } \\
4-12 \text { min nach Applikation }\end{array}$ \\
\hline Nebenwirkungen & $\begin{array}{l}\text { - Bradykardie } \\
\text { - Blutdruckabfall } \\
\text { - Schwindel } \\
\text { - Kopfschmerzen }\end{array}$ & $\begin{array}{l}\text { - hypotone Kreislaufzustände durch } \\
\text { Senkung des Blutdrucks } \\
\text { - Kopfschmerzen } \\
\text { - Reflextachykardie }\end{array}$ \\
\hline
\end{tabular}

\ Tab. 1.3 Prämedikation und ihre Kontraindikationen.

Betablocker (z. B. Metoprolol)

dekompensierte Herzinsuffizienz

Asthma bronchiale

systolischer Blutdruck $<100 \mathrm{mmHg}$

Einnahme von Medikamenten, welche die atrioventrikuläre Überleitungszeit verlängern (z. B. Verapamil, Diltiazem, Digoxin ...)

\section{Nitroglycerin}

schwere Aortenklappeninsuffizienz

hypertrophe obstruktive Kardiomyopathie

hypotone Kreislaufzustände

Einnahme von Phosphodiesteraseinhibitoren (z. B. Sildenafil -

Viagra ${ }^{\circledR}$ bei erektiler Dysfunktion oder pulmonaler Hypertonie)

bekannte Allergie gegen $\beta$-Rezeptor-Antagonisten

Herzens regelmäßig sein. Nitroglycerin wirkt gefäßerweiternd und sorgt somit für eine verbesserte Darstellung auch kleinerer Koronararterien.

Der geringe Gefäßdurchmesser der Koronararterien setzt für eine gute Beurteilbarkeit eine rasche und hohe Kontrastmittelkonzentration voraus. Um das Kontrastmittel mit einem ausreichenden Fluss zu injizieren, sollte möglichst ein 18G-Venenverweilkatheter verwendet werden. Dieser sollte möglichst schon vor Betreten des eigentlichen Untersuchungsraumes, vorzugweise in der rechten Ellenbeuge, platziert worden sein.

Ein Einüben des Atemkommandos mit dem Patienten ist essenziell für die Minimierung von Bewegungsartefakten während der Aufnahme der CT-Bilder. Während des Atemkommandos sollten mögliche EKG-Veränderungen oder ein Ansteigen der Herzfrequenz beobachtet werden. Gegebenenfalls muss auf ein anderes Scanprotokoll zurückgegriffen werden. Die Koronar-CT sollte in mittle- rer Inspirationslage ohne Pressen durchgeführt werden. Die koronare CT sollte nicht durchgeführt werden, wenn der Patient Atemkommandos nicht befolgen kann.

Merke

Ein Einüben des Atemkommandos mit dem Patienten ist essenziell für die Minimierung von Bewegungsartefakten während der Aufnahme der CT-Bilder.

\section{Lagerung des Patienten - EKG-Anlage}

Die EKG-Anlage erfolgt nach Angaben des Herstellers und sollte möglichst außerhalb des Scanbereichs angebracht werden, um Fehler und Bildartefakte zu vermindern. Die entsprechenden Hautareale sollten vorher gereinigt und Behaarung ggf. durch Rasur entfernt werden. Der Patient wird in der Regel in Rückenlage auf dem Untersuchungstisch positioniert, beide Arme liegen oben hinter dem Kopf. Bei Patientinnen mit großen Mammae kann ein Hochbinden zur Senkung der Brustdosis sinnvoll sein [12]. Das Herz sollte in der Mitte der Gantry positioniert werden, 
d. h. der Patient nicht in der Mittellinie, sondern etwas nach rechts versetzt liegen. Die seitliche Positionierung der optimalen Tischhöhe ist nicht ganz so einfach. Die Mitte des Herzens projiziert sich in der Seitansicht auf die obere Drittellinie [13]. Dies ist wichtig für eine gute Bildqualität und eine evtl. Anwendung einer Dosismodulation.

\section{Auswahl des Scanprotokolls}

Grundsätzlich kann zwischen 2 typischen Scanmodi unterschieden werden:

- dem prospektiv-axialen Scanmodus, bei welchem eine sequenzielle Untersuchung in der gewünschten Herzphase durchgeführt wird, mit Tischvorschub zwischen den einzelnen Scans.

- dem Spiral-CT mit retrospektivem EKG-Gating. Dabei wird der Tisch kontinuierlich bewegt und Bilddaten der gesamten Herzaktion erfasst.

Beim retrospektiven Gating kann jede erwünschte Phase des Herzzyklus zur Bildrekonstruktion herangezogen werden und damit auch funktionelle Untersuchungen und die Erzeugung bewegter Bilder ermöglichen. Vor- und Nachteile sind in $>$ Tab. 1.4 zusammengefasst.

Scanner im High-End-Segment weisen bezüglich der möglichen Scanmodi Besonderheiten auf:

- Sogenannte Volumen-CT (z. B. GE Revolution, Toshiba Aquilion ONE) mit 16 cm Detektorbreite ermöglichen die Erfassung des gesamten Herzens im prospektiven Scanmodus ohne jeglichen Tischvorschub.

- Bei Dual-Source-Scannern (Siemens Definition Flash/Force) mit 2 Röntgenröhren kann mit hohen Pitch-Werten (bis 3,4) das ganze Herz in einem schnellen Spiralscan von ca. 400 ms erfasst werden (sog. „Flash-Modus“). Dies setzt in der Regel eine besonders niedrige und regelmäßige Herzfrequenz voraus.
Durch die Entwicklung von Dual-Source-CT und WideDetector-Scannern ist mittlerweile eine Herzbildgebung von Patienten mit hoher und irregulärer Herzfrequenz oder sogar Vorhofflimmern mit diagnostischer Bildqualität möglich.

In der Regel bewegt sich das Herz in der Herzdiastole (Herzfüllungsphase) am wenigsten. Dies ist vor allem für die Koronararteriendarstellung wichtig. Bei niedriger und regelmäßiger Herzfrequenz ist eine Bildakquisition in der mittleren Diastole (etwa von 60-80 \% des RR-Zyklus) meist ausreichend. Bei höheren Herzfrequenzen oder Arrhythmien kann eine Ausweitung auf die Endsystole nötig sein - z. B. 30-80 \% des RR-Zyklus, oder alternativ bei Dual-Source-CTs ein rein endsystolischer Scan bei 200400 ms nach der R-Zacke.

Je nach Scanmodus und Scannertyp können die mit voller Dosis abgebildeten Herzphasen automatisch oder manuell angepasst werden. Um beim retrospektiven Gating Dosis zu sparen, können die nicht unbedingt benötigten Herzphasen mit geringerem Röhrenstrom, aber damit auch höherem Bildrauschen (EKG-basierte Dosismodulation), aufgenommen werden. Eine Auswertung der Ventrikelfunktion ist damit aber dennoch möglich ( $\mathbf{A b b}$. 2). Im prospektiven oder „Step-and-Shoot“-Scanmodus hingegen werden ausschließlich die gewählten Herzphasen erfasst.

Die Auswahl der Dosisparameter (Röhrenspannung und Röhrenstrom) erfolgt entweder manuell nach Körpermaßen oder durch eine Dosisautomatik (Dosisparameterberechnung anhand des Topogramms). Generell wird beim Koronar-CT eine möglichst niedrige Röhrenspannung (möglichst 100 kV für BMI < 25-30; darüber 120 kV) angestrebt, um einen guten Jodkontrast zu erreichen.

- Tab. 1.4 Vor- und Nachteile prospektiven und retrospektiven Gatings.

\begin{tabular}{|c|c|c|}
\hline & Prospektives Gating & Retrospektives Gating \\
\hline Vorteile & $\begin{array}{l}\text { Patienten werden nur während der kurzen Messphase } \\
\text { „bestrahlt“ - somit geringere Strahlenexposition }\end{array}$ & $\begin{array}{l}\text { - kontinuierliche Erfassung der gesamten Herzaktion } \\
\text { - Daten können aus jeder Phase des Herzzyklus zur } \\
\text { Bildrekonstruktion verwendet werden } \\
\text { - sehr flexible Methode auch bei schwierigen Patientenver- } \\
\text { hältnissen durch retrospektive Optimierung der } \\
\text { Rekonstruktionsphasen } \\
\text { - Aussagen über ventrikuläre und valvuläre Funktion } \\
\text { möglich }\end{array}$ \\
\hline Nachteile & $\begin{array}{l}\text { - wegen diskontinuierlicher Tischbewegung kann in } \\
\text { der Regel nur jeder 2. Herzschlag für die Bildgebung } \\
\text { genutzt werden } \\
\text { - Verfahren weniger flexibel - eine spätere Rekonst- } \\
\text { ruktion der Bilder zur Optimierung der Bildqualität } \\
\text { nur bedingt möglich } \\
\text { - keine Aussage über Herzfunktion möglich }\end{array}$ & $\begin{array}{l}\text { - Anpassung des Tischvorschubs an die Herzfrequenz des } \\
\text { Patienten zur Vermeidung von Abtastlücken nötig } \\
\text { - höhere Strahlenexposition durch „Bestrahlung“ des } \\
\text { Patienten während der gesamten Aufnahmezeit }\end{array}$ \\
\hline Anwendung & $\begin{array}{l}\text { Patienten mit niedriger und regelmäßiger Herzfre- } \\
\text { quenz }\end{array}$ & $\begin{array}{l}\text { - Patienten, bei welchen eine rhythmische Herzfrequenz } \\
\text { nicht garantiert werden kann } \\
\text { - Patienten mit zu schneller Herzfrequenz }\end{array}$ \\
\hline
\end{tabular}




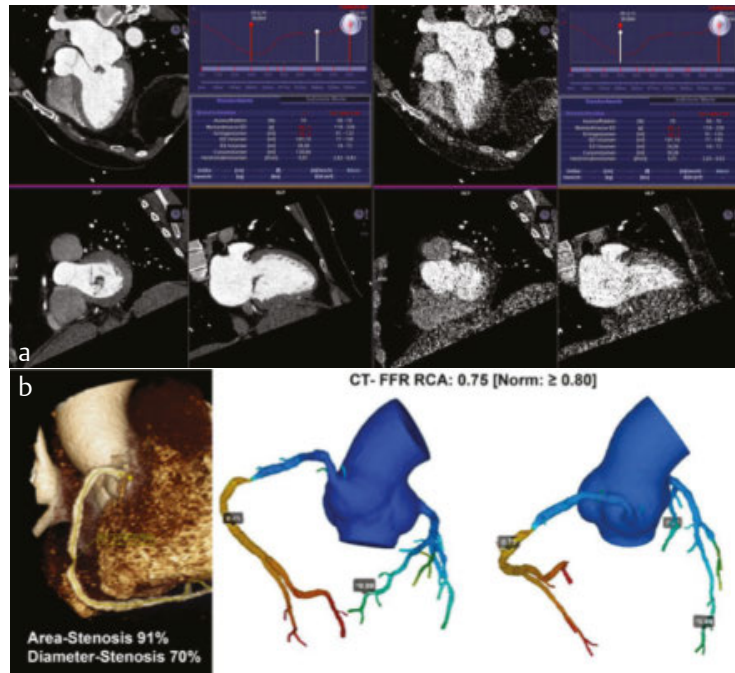

-Abb. 2 Koronar-CT und Darstellung der rechten Koronararterie. a Retrospektiv gegatetes Koronar-CT mit Dosismodulation (Reduktion des Röhrenstroms in der Systole - Siemens MinDose) bei einem 59-jährigen Patienten mit Mitralklappenendokarditis nach Mitralklappenrekonstruktion. Deutlich zu erkennen ist in der rechten Bildhälfte das verstärkte Rauschen in der Systole durch Absenkung des Röhrenstroms. Die in der Diastole (linke Bildhälfte) rekonstruierten Schichten zeigen ein geringes Rauschen und lassen die endokarditische Vegetation an der anterioren Mitralklappe gut erkennen. b VolumeRendering (VRT)-Darstellung der rechten Koronararterie desselben Patienten wie in a mit Nachweis einer morphologisch hochgradigen Stenose im mittleren Drittel und korrespondierende Bestimmung der CT-FFR, die eine Reduzierung und damit eine hämodynamische Relevanz nachweist $(<0,80)$.

\section{HINTERGRUNDWISSEN}

Ausgeprägte Arrhythmien bzw. Vorhofflimmern sind relative Kontraindikationen für ein Koronar-CT. Wird dennoch ein Koronar-CT durchgeführt, wird üblicherweise ein retrospektives Gating ohne Dosismodulation verwendet, um die Rekonstruktion aller Herzphasen und eine evtl. Editierung der EKG-Synchronisationspunkte zu ermöglichen. Dies ist allerdings mit einer hohen Strahlenexposition verbunden.

Aufgrund der verschiedenen Scantechniken und zahlreichen Einstellmöglichkeiten kann ein Koronar-CT eine sehr unterschiedliche effektive Patientendosis von $<1 \mathrm{mSv}$ bis $15 \mathrm{mSv}$ und mehr mit sich bringen [14]. Die diagnostischen Referenzwerte des Bundesamtes für Strahlenschutz von 2016 betragen für ein Koronar-CT im prospektiven Scanmodus für $\mathrm{CTDI}_{\text {vol }}{ }^{10} 20 \mathrm{mGy}$ und für DLP10 330 mGy*cm [15].
Adaptation der Protokolle bei übergewichtigen Patienten Durch eine höhere Streustrahlung im Körper eines adipösen Patienten entsteht ein niedrigeres Signal-RauschVerhältnis. Bei der Herz-CT steigt dadurch die Rate der Koronarsegmente mit nicht diagnostischer Bildqualität.

Das erhöhte Bildrauschen kann durch dickere Schichten, breitere Detektorkollimation und langsamere Rotationszeiten minimiert werden. Die Bildqualität bei adipösen Patienten profitiert grundsätzlich von einem erhöhten Röhrenstromzeit-Produkt und/oder erhöhter Röhrenspannung. Bei adipösen Patienten mit einem Body-MassIndex (BMI) $>30$ kg / qm ist die Erhöhung der Röhrenspannung höchst effektiv. Die Anpassung der Röhrenspannung auf 140 kV liefert eine diagnostische Bildqualität auch bei Patienten mit einem BMI zwischen 30 und 35 kg / qm.

\section{Durchführung des Scans}

Nach der korrekten Lagerung, Prämedikation und Auswahl des Scanprotokolls beginnt nun die eigentliche Bildaufnahme. Typischerweise startet die Durchführung mit einer Übersichtsaufnahme (Topogramm) a. p. ggf. auch lateral. Es folgt die Einstellung des Field of View (FOV) und Festlegung der Scanlänge ( $\triangleright$ Abb. $\mathbf{3})$. Während für ein normales Koronar-CT die Abdeckung von der Trachealbifurkation bis zum Herzunterrand (im Bild grün) ausreichend ist, kann z. B. für die Darstellung von Bypässen aus der Aorta (orange) oder der A. thoracica interna (rot) eine größere Scanlänge erforderlich sein.

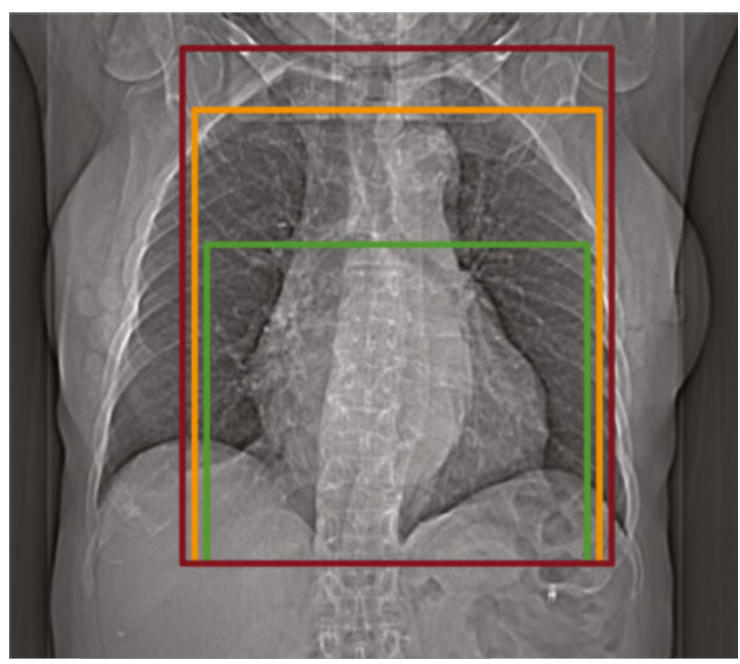

Abb. 3 Scanlängen für Koronar- (grün) bzw. Bypass-(orange) CT.

Gegebenenfalls kann vor dem eigentlichen Scan noch ein natives Kalzium-Scoring ausgeführt werden. Hier kann die koronare Kalklast quantifiziert werden. Außerdem können die Herzgrenzen eindeutiger festgelegt werden. Eventuell wird bei zu hoher Kalklast bzw. schwerer zirkulärer Verkalkung eines Koronarsegmentes ( $\triangleright$ Abb. 4) die Untersuchung an dieser Stelle nicht weiter fortgesetzt. 


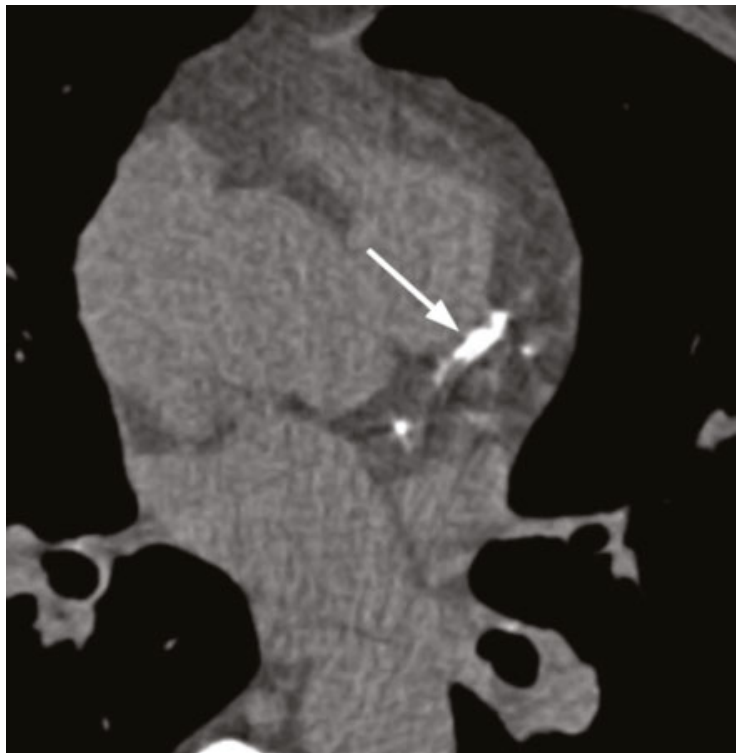

-Abb. 4 Natives Kalzium-Scoring mit schwerer Verkalkung der mittleren LAD (Pfeil).

Für den Scanstart kann ein Testbolus- oder Bolustrackingverfahren eingesetzt werden (Vor- und Nachteile siehe - Tab. 1.5), fixe Delays sind insbesondere bei Herzpatienten weniger geeignet [11]. Der Scan für das Prämonitoring erfolgt typischerweise auf Höhe der Trachealbifurkation. Anschließend wird die Region of Interest (ROI) in der Aorta ascendens positioniert und der Scan ausgelöst. Bei Volumen- bzw. Dual-Source-CT wird eventuell eine ROI bereits im linken Vorhof oder der linken Herzkammer gewählt.

Bei der Kontrastmittelapplikation sollte ein bi- oder triphasisches Protokoll angewendet werden.

Wird die Flussrate und das Volumen sowohl an die Körpermaße des Patienten als auch an die Scanzeit angepasst, so erhält man eine gute Kontrastierung. Die Anpassung kann mittels Tabellen oder kommerzieller Software vorgenommen werden. Das Kontrastmittelvolumen wird im Allgemeinen wie folgt berechnet:

Scanzeit $[\mathrm{s}]+$ Delay $[\mathrm{s}]$ x Flussrate $[\mathrm{ml} / \mathrm{s}]=$ Kontrastmittelvolumen $[\mathrm{ml}]$

Eine Beispieltabelle unter Verwendung eine Kontrastmittelkonzentration von $370 \mathrm{mg} / \mathrm{ml}$ ist in Tab. $\mathbf{1 . 6}$ dargestellt.

\section{Bildrekonstruktion und Postprocessing}

Aus dem akquirierten Datensatz werden axiale Bildrekonstruktionen mit einer Schichtdicke $<1 \mathrm{~mm}$ überlappend

- Tab. 1.5 Vor- und Nachteile verschiedener Methoden zum Scan-Timing

\begin{tabular}{|c|c|c|c|}
\hline & Fixes Zeitintervall & Test-Bolus & Bolus-Tracking \\
\hline Technik & $\begin{array}{l}\text { fixes festgelegtes Zeitintervall } \\
\text { zwischen Kontrastmittelapplikation } \\
\text { und Beginn Bildaufnahme }\end{array}$ & $\begin{array}{l}\text { Bestimmung der Transitzeit des } \\
\text { Kontrastmittels anhand Anreiche- } \\
\text { rungskurve }\end{array}$ & $\begin{array}{l}\text { Monitoring der arteriellen } \\
\text { Kontrastmittelanreicherung in } \\
\text { Echtzeit }\end{array}$ \\
\hline Vorteil & Gute Standardisierung & $\begin{array}{l}\text { gezielte Anpassung der Kontrastmit- } \\
\text { telgabe an die Transitzeit des } \\
\text { Patienten }\end{array}$ & $\begin{array}{l}\text { einfach und schnell } \\
\text { Einsparung von Kontrastmittel } \\
\text { geringere Anfälligkeit bei Arhythmien }\end{array}$ \\
\hline Nachteil & $\begin{array}{l}\text { für gute Kontrastierung des Herzens } \\
\text { nicht geeignet }\end{array}$ & $\begin{array}{l}\text { höhere Kontrastmitteldosis als bei } \\
\text { Bolustracking }\end{array}$ & $\begin{array}{l}\text { exakte anatomische Positionierung } \\
\text { der Region of interest (ROI) notwendig }\end{array}$ \\
\hline
\end{tabular}

- Tab. 1.6 Beispiel eines patienten- und scanzeitadaptierten Kontrastmittelprotokolls.

\begin{tabular}{|c|c|c|c|c|}
\hline & BMI & $<25$ & $25-30$ & $>30$ \\
\hline & Fluss/Scanzeit & $4,5 \mathrm{ml} / \mathrm{s}$ & $5 \mathrm{ml} / \mathrm{s}$ & $5,5 \mathrm{ml} / \mathrm{s}$ \\
\hline \multirow[t]{8}{*}{ 1. Phase } & $5 \mathrm{~s}$ & $45 \mathrm{ml}$ & $50 \mathrm{ml}$ & $55 \mathrm{ml}$ \\
\hline & $6 s$ & $50 \mathrm{ml}$ & $55 \mathrm{ml}$ & $61 \mathrm{ml}$ \\
\hline & $7 \mathrm{~s}$ & $54 \mathrm{ml}$ & $60 \mathrm{ml}$ & $66 \mathrm{ml}$ \\
\hline & $8 \mathrm{~s}$ & $59 \mathrm{ml}$ & $65 \mathrm{ml}$ & $72 \mathrm{ml}$ \\
\hline & $9 \mathrm{~s}$ & $63 \mathrm{ml}$ & $70 \mathrm{ml}$ & $77 \mathrm{ml}$ \\
\hline & $10 \mathrm{~s}$ & $68 \mathrm{ml}$ & $75 \mathrm{ml}$ & $83 \mathrm{ml}$ \\
\hline & $11 \mathrm{~s}$ & $72 \mathrm{ml}$ & $80 \mathrm{ml}$ & $88 \mathrm{ml}$ \\
\hline & $12 \mathrm{~s}$ & $77 \mathrm{ml}$ & $85 \mathrm{ml}$ & $94 \mathrm{ml}$ \\
\hline 2. Phase & \multicolumn{4}{|c|}{ gleiches Volumen mit 30:70 (Kontrastmittel:NaCl) } \\
\hline 3. Phase & \multicolumn{4}{|l|}{$50 \mathrm{ml} \mathrm{NaCl}$} \\
\hline
\end{tabular}



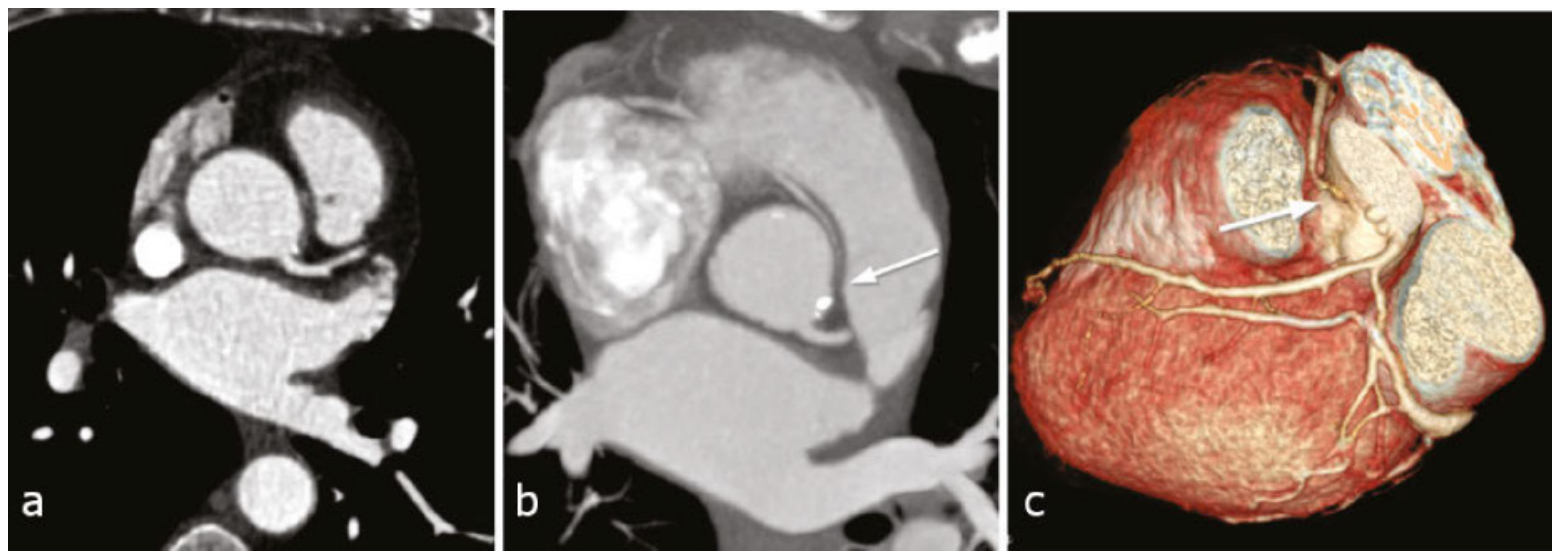

-Abb. 5 Koronar-CT mit atypischem Abgang der rechten Koronararterie (Pfeile).

a Darstellung in axialer Primarrekonstruktion.

b Darstellung in gewinkelter MIP.

c 3-D-Rekonstruktion.

berechnet ( $\triangleright$ Abb. 5). Ein FOV von $\leq 200-250 \mathrm{~mm}$ sorgt für eine gute Ortsauflösung. Für die Rekonstruktion werden Gefäßkernel verwendet. Bei Kalk oder Stents finden härtere Kernel Anwendung. Durch einen iterativen Rekonstruktionsalgorithmus kann das Bildrauschen vermindert werden, wodurch eine Dosisminderung von 20-70\% erreichbar ist.

Die Bildrekonstruktion ist von der Bewegung der Koronararterien während eines Herzzyklus und der Veränderung der Herzphasendauer je nach Herzfrequenz abhängig. In der frühen Systole (0-20\% des RR-Intervalls), frühen Diastole (40-50 \% des RR-Intervalls) und späten Diastole (80-90 \% des RR-Intervalls) zeigen nahezu alle Koronarsegmente eine schnelle Bewegung, wohingegen alle Koronarsegmente in der mittleren Diastole (55-65 \% des RR-Intervalls) ein Bewegungsminimum und eine zweite relative Ruhephase in der Endsystole (25-35\% des RRIntervalls) aufweisen.

\section{HINTERGRUNDWISSEN}

Die Mittdiastole ist bei niedriger Herzfrequenz am besten zur CT-Rekonstruktion geeignet. Mit zunehmender Herzfrequenz verkürzen sich Systole und Diastole ungleichmäßig. Die Systolendauer nimmt zwischen 60 bpm und 200 bpm um etwa die Hälfte ab - die Diastole verkürzt sich um 80 \%. Mit steigender Herzfrequenz ist die endsystolische Datenrekonstruktion häufig mit besseren Ergebnissen assoziiert als die mittdiastolische Rekonstruktion.

Maximale Intensitätsprojektionen und 3-D-RenderingDarstellungen (Abb. 5) erleichtern insbesondere den Überblick über die Anatomie, wie hier bei atypischem Abgang der rechten Koronararterie von links (Pfeile).

\section{FALLBEISPIEL}

Neben gewöhnlichen sagittalen und koronaren multiplanaren Reformatierungen (MPR) kommen auch gekrümmte MPR (Curved MPR) entlang der Koronargefäße zum Einsatz. In \ Abb. 6 sind auf diese Weise die Herzkranzgefäße eines 71-jährigen männlichen Patienten mit Belastungsdyspnoe dargestellt. Im linken absteigenden Koronarast (Left anterior Descending, LAD) fand sich ein teilweise verkalkter Plaque mit hochgradiger Stenose > 70 \%. In der nachfolgenden Herzkatheteruntersuchung konnte diese bestätigt und mit Ballonangioplastie und Stent versorgt werden ( $\triangleright$ Abb. 7).

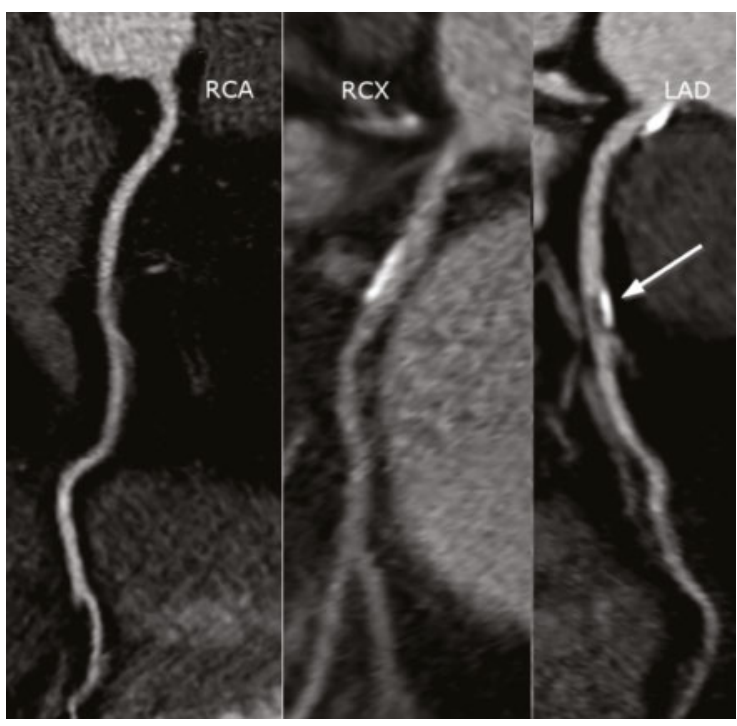

-Abb. 6 Gekrümmte Reformatierungen (Curved MPR) der Herzkranzgefäße. Relevante Stenose der linken absteigenden Koronararterie (Pfeil). LAD = Left anterior Descending; RCA = Right coronary Artery; $R C X=R$. circumflexus. 


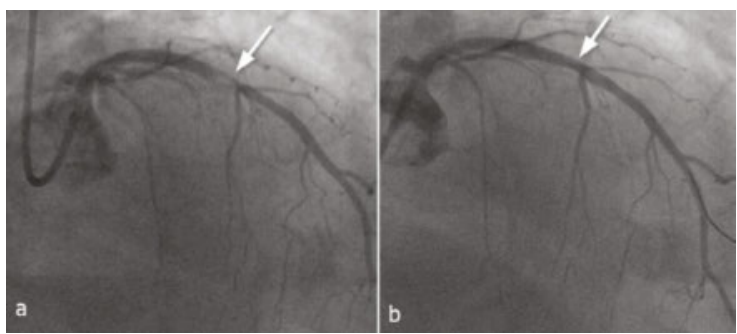

Abb. 7 Gleicher Patient wie in Abb. 6.

a Bestätigung der hochgradigen Koronarstenose.

b Ergebnis nach Angioplastie und Stentimplantation.

Ausblick

Während die Koronar-CT zum Ausschluss von Koronarstenosen eine exzellente Genauigkeit mit einem negativen prädiktiven Wert von bis zu 98\% aufweist [16], ist sie weniger gut zum Beweis einer hämodynamischen Relevanz einer Stenose geeignet. Um diese Lücke zu schließen, werden insbesondere zwei zusätzliche Techniken diskutiert [17]:

- Die CT-basierte fraktionelle Flussreserve (FFR-CT), die die invasive Kathetermessung simuliert: Hier wird auf Basis eines normalen CT-Datensatzes unter Einsatz flussdynamischer Modelle ein FFR-Wert berechnet (Abb. 2), welche in einigen Studien sehr gut mit der Kathetermessung korreliert [18].

- Die CT-Perfusion unter Adenosin- oder Regadenosonstress: Durch intravenöse Gabe eines Vasodilatators wird eine relative Minderdurchblutung von Arealen mit relevanten Koronarstenosen provoziert, welche visuell oder quantitativ beurteilt werden kann.

\section{Stress-MRT}

\section{Patientenvorbereitung und Patientenaufklärung}

In Vorbereitung auf die Stress-MRT des Herzens muss eine sehr genaue, ausführliche und intensive Aufklärung erfolgen. Der Patient sollte über die Nutzen und Risiken der Perfusionsuntersuchung aufgeklärt werden und sein schriftliches Einverständnis geben.

Im Zusammenhang mit Adenosin bzw. Regadenoson müssen

- methylxanthinhaltigen Nahrungsmittel wie

- Kaffee

- Tee

- Cola

- Schokolade

- Energydrinks

- theophyllinhaltige Medikamente

- Medikamente, die die AV-Überleitungszeit erhöhen

- Betablocker

mindestens 6-12 h, besser $24 \mathrm{~h}$ vor der Untersuchung abgesetzt bzw. nicht mehr eingenommen werden, da sie die Adenosinwirkung unspezifisch antagonisieren und damit die Auslösung der Gefäßdilatation verhindern bzw. vermindern können. Der Patient muss über mögliche Nebenwirkungen des Stressmedikaments durch einen Arzt aufgeklärt werden. Die wichtigsten Kontraindikationen und Nebenwirkungen für eine Adenosin- bzw. Dobutaminbelastung sind in Tab. > Tab. 1.7 aufgelistet.

\section{HINTERGRUNDWISSEN}

Der Wirkmechanismus der über den Adenosinrezeptor wirkenden Medikamente (Adenosin, Regadenoson) mit einer Gefäßerweiterung der gesunden Gefäße und einem entsprechenden „Steal Effect“ aus den erkrankten Gefäßen ist ein völlig anderer als der von Dobutamin, bei dem es als direkte Wirkung des Medikaments zu einer Herzfrequenz- und Kontraktilitätssteigerung kommt.

Über den Adenosinrezeptor wirkende Medikamente sind besonders für die Beurteilung der Herzdurchblutung unter Belastung geeignet. Die Steigerung der Herzrate bei Adenosineinsatz erfolgt nicht direkt, sondern über eine sog. Reflextachykardie und kann anders als beim Dobutamin deshalb nur bedingt als Wirkkontrolle des Medikamentes genutzt werden.

Dobutamin wird vor allem in der Echokardiografie eingesetzt und steigert über ß1-Rezeptoren direkt die Herzfrequenz und die Kontraktilität. In der Stress-MRT findet Dobutamin nur noch selten Verwendung (ca. 3 \% aller Stressuntersuchungen nach dem ESCR MRCT-Registry - Stand 01.01.2017 [9]), vor allem bei Kontraindikationen für die Verwendung von Adenosin (z. B. Asthma bronchiale). Hier wird dann eine neu aufgetretene oder verstärkte Wandbewegungsstörung unter Dobutaminbelastung als „Ischämiemarker“ interpretiert.

\section{Lagerung und relevante Maßnahmen am Patienten}

Wie bei anderen MRT-Untersuchungen sollte auch bei der Stress-MRT auf eine möglichst bequeme Lagerung des Patienten geachtet werden. Die Lagerung erfolgt in Rückenlage und kann durch eine Knierolle angenehmer gestaltet werden. Die Arme des Patienten können beidseits neben dem Körper liegen.

Bei der Stress-MRT sind in der Regel 2 Venenverweilkanülen notwendig. Die größere wird für die Kontrastmittelgabe verwendet, die kleinere für die Gabe des Stressmedikamentes.

Eine adäquate Überwachung des Patienten während der Untersuchung ist unabdingbar. Die Vitalparameter wie Herzfrequenz, Blutdruck und ggf. nicht invasive Sauerstoffsättigung über eine Pulsoxymetrie sollten während der Belastungs- und Erholungsphase vom Arzt erfasst und in einem Belastungsprotokoll dokumentiert werden. Die Amplitude der Pulsoxymetrie kann auch zur Triggerung des Scans und zur Überwachung der Herzaktionen genutzt werden. 
> Tab. 1.7 Wirkung, Kontraindikationen, mögliche Komplikationen Adenosin versus Dobutamin [modifiziert nach [18].

\begin{tabular}{|c|c|}
\hline Adenosin (gilt mit Einschränkungen auch für Regadenoson) & Dobutamin \\
\hline \multicolumn{2}{|l|}{ Wirkmodus } \\
\hline $\begin{array}{l}\text { auf Adenosinrezeptoren (A1, A2b, A3) } \\
\rightarrow \text { Vasodilatation }\end{array}$ & $\begin{array}{l}\text { auf ß1-Rezeptoren } \\
\rightarrow \text { Kontraktilitätssteigerung } \\
\text { (geringer Vasodilatation ß2) }\end{array}$ \\
\hline \multicolumn{2}{|l|}{ Voraussetzung für Anwendung } \\
\hline \multicolumn{2}{|l|}{$\begin{array}{l}\text { Verzicht auf methylxanthinhaltige Nahrungsmittel: wie Kaffee, } \\
\text { Teekonsum, Schokolade, Energydrinks mindestens für 6-12 h, } \\
\text { besser } 24 \text { h pausieren! }\end{array}$} \\
\hline \multicolumn{2}{|l|}{ Nebenwirkungen } \\
\hline$\downarrow$ RR-Abfall $(8-10 \mathrm{mmHg})$ & Tachykardie \\
\hline Reflextachykardie & Kammerflimmern und Kammerflattern (<0.1\%) \\
\hline AV-Block (I. $\left.10 \% ;>1 .^{\circ} 5 \%\right)$ & $\begin{array}{l}\text { ischämische Wandbewegungsstörungen mit Reduktion der EF } \\
\text { oder Anstieg des ESV }\end{array}$ \\
\hline Dyspnoe & Palpitationen \\
\hline Brustschmerz via A1-Rezeptor =Schmerzrezeptoren & Hypotension (-10 \%) \\
\hline \multicolumn{2}{|l|}{ Kontraindikationen } \\
\hline instabile Angina pectoris & instabile Angina pectoris \\
\hline akuter Myokardinfarkt & akuter Myokardinfarkt \\
\hline schweres Asthma (relativ COPD) & $\begin{array}{l}\text { schwere Aortenklappenstenose, } \\
\text { schwere HOCM }\end{array}$ \\
\hline
\end{tabular}

\section{HINTERGRUNDWISSEN}

Die Blutdruckmanschette sollte bei Adenosin- und Dobutaminbelastung nicht auf der Seite der kontinuierlichen intravenösen Medikamenteninfusion, sondern auf der Seite der Kontrastmittelapplikation angebracht werden. Besser manueller als automatischer Start der Messung, damit sich die Manschette nicht gerade währen der Kontrastmittelgabe aufpumpt. Regadenoson kann im „Bolus“ über 10 s ohne 2 . Venenverweilkanüle appliziert werden.

Das EKG sollte entsprechend der Anweisungen des Geräteherstellers geklebt werden. Es sind dafür spezielle MR-kompatible Elektroden zu verwenden. Auf einen guten Hautkontakt ist zu achten, ggf. muss die Behaarung entfernt und die Haut entfettet werden. Es sollte die EKG-Ableitung mit der höchsten R-Zacken-Amplitude gewählt werden. Auf jeden Fall muss die Amplitude der RZacke deutlich höher als die der T-Welle sein. Bei schlechter EKG-Ableitung kann der Perfusionsscan auch über die Pulsoxymetrie getriggert werden. Um in der Diastole zu messen, muss dann nur das Triggerdelay entsprechend angepasst werden.

\section{Merke}

Es hat sich bewährt, mit dem Patienten die Atemkommandos vorher zu üben. Die MTRA sollte die Atemkommandos an den Atemrhythmus des Patienten anpassen und ausreichend Atemerholungsphasen lassen.

\section{Auswahl des Scanprotokolls - Adenosinstress versus Dobutaminstress}

In der Stress-MRT werden zur pharmakologischen Belastung vor allem Medikamente, die über den Adenosinrezeptor wirken (Adenosin, Regadenoson) eingesetzt. Diese erlauben die Beurteilung einer Herzmuskeldurchblutungsstörung durch eine direkte, meist visuelle oder semiquantitative Beurteilung der Myokardperfusion. Das früher vor allem in der Myokardszintigrafie häufig eingesetzte Dipyridamol führt indirekt über eine Steigerung der Adenosinkonzentration im Blut zu einer Belastungssituation, spielt aber in der Stress-MRT in Deutschland keine Rolle mehr.

Für die MR-Perfusionsanalyse werden kontrastmittelgestützte schnelle T1-gewichete Gradientenecho- (GRE) oder Hybrid-Echo-Planar-Imaging (EPI)-Sequenzen zur „First-Pass“-Analyse, d. h. zur Analyse des ersten Kontrastmitteldurchgangs, verwendet. Deshalb müssen die 
Einzelbilder möglichst schnell aufgenommen werden (üblicherweise drei repräsentative Bilder pro Sekunde). Im Vergleich zu den GRE-Sequenzen sind die EPI-Sequenzen etwas schneller. Beide weisen ein gutes SignalRausch-Verhältnis auf. Durch Verwendung von SSFP-Gradientenechosequenzen, durch Sättigungs-Vorpulse oder durch Scannen bei höherer Feldstärke (Untersuchung bei 3,0 anstelle von 1,5 T) können sowohl das Signal-Rauschals auch das Kontrast-Rausch-Verhältnis weiter verbessert werden. Insbesondere für die meist rein visuelle Auswertung ist dies von Vorteil ( $\triangleright$ Abb. 8).
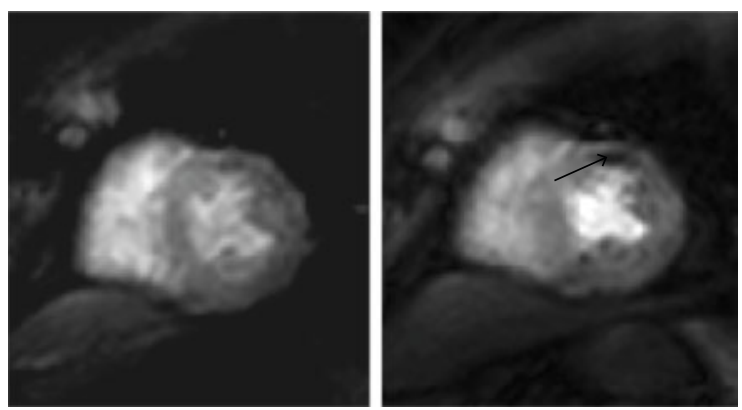

-Abb. 8 Mittventrikulärer MR-Kurzachsenschnitt des linken Ventrikels einer „First-Pass“-Perfusionsanalyse desselben Patienten unter Adenosinstress bei 1,5 T (links) und 3,0 T (rechts). Der kleine belastungsinduzierte Perfusionsdefekt im Bereich der linksventrikulären Vorderwand (Pfeil) war nur bei 3 T eindeutig nachweisbar.

Eine Abdeckung mit drei repräsentativen Kurzachsenschnitten über den linken Ventrikel ist in der Regel ausreichend. Im Mittel sollten für eine MR „First-Pass“-Perfusionsanalyse pro Schicht 60 Bilder akquiriert werden. Die Messdauer und „Atem-Anhalte-Phase“ kann deshalb bis zu 60 s dauern. Es empfiehlt sich deshalb, ein zweigeteiltes Atemkommando

- kurz vor Start der Messung

- kurz vor der Ankunft des Kontrastmittelbolus durchzuführen.

\section{HINTERGRUNDWISSEN}

Das Adenosin wird üblicherweise in einer gewichtsangepassten Dosierung von $140 \mathrm{\mu g} / \mathrm{kg}$ Körpergewicht/min i. v. über 6 min eingesetzt. Nach 3 min und Erreichen eines pharmakologischen Gleichgewichtes („Steady State“) beginnt die Kontrastmittelgabe in einer Dosierung von üblicherweise 0,05-0,1 mmol / kg Körpergewicht mit einer Flussrate von 3-7 ml/ s.

Die Applikation von Regadenoson in Hinblick auf Vorbereitung und Dauer ist deutlich einfacher und schneller als das von Adenosin und wird mittlerweile in bis zu 6 \% (vs. 91 \% Adenosin) aller Stress-MR-Untersuchungen [9] verwendet ( $\triangleright$ Abb. 9). Der selektive Adenosinrezeptoragonist Regadenoson kann ohne Gewichtsadaptation als Bolus (5 ml) über $10 \mathrm{~s}$ in immer gleicher Dosierung von $400 \mu \mathrm{g}$ (Rapiscan) i. v. appliziert werden, gefolgt von $5 \mathrm{ml}$ einer Natriumchloridinjektionslösung $9 \mathrm{mg} / \mathrm{ml}(0,9 \%)$. Als selektiver $\mathrm{A}_{2 \mathrm{~A}}$-Adenosinrezeptoragonist weißt Regadenoson insgesamt geringere Nebenwirkung auf. Für die „First-Pass“Perfusionsanalyse kann die MR-Messung mit Kontrastmittel bereits 10-20 s nach der $\mathrm{NaCl}$-Gabe erfolgen.

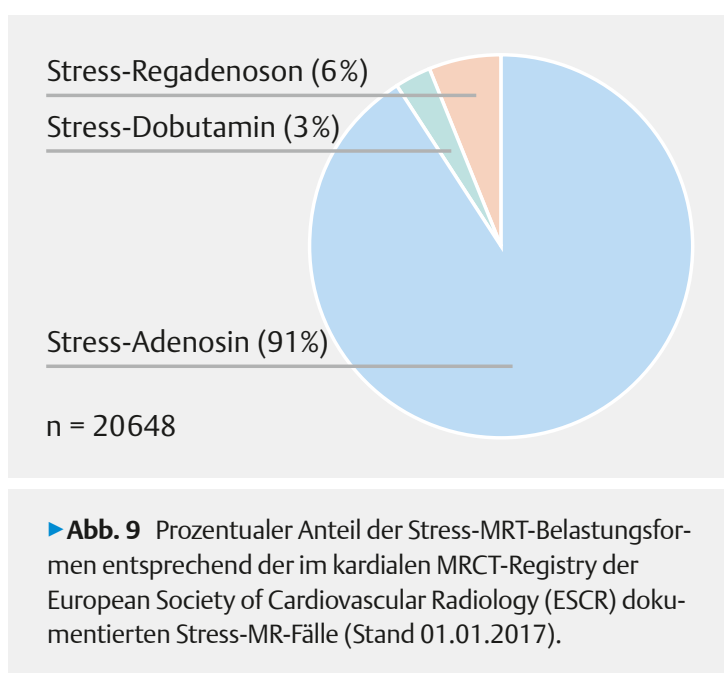

Merke

Bei Gabe von Adenosin, aber vor allem bei Gabe von Regadenoson muss immer der Antagonist Theophyllin i. v. als Notfallmedikament bereitgehalten werden.

Bei Dobutaminstress wird im Gegensatz zum Adenosin der über ß1-Rezeptoren vermittelte positiv inotrope (kontraktilitätssteigernde) und positiv chronotrope (herzfrequenzsteigernde) Effekt ausgenutzt, der im nicht pathologischen Fall über einen verstärkten Sauerstoffbedarf auch zu einer Steigerung der myokardialen Perfusion führt. Ziel der Dobutamin-Stressuntersuchung ist allerdings vor allem der Nachweis belastungsbedingter neu aufgetretener oder unter Belastung verstärkter myokardialer Wandbewegungsstörungen.

Merke

Bei Verwendung eines sog. „Low-Dose“ (5-10 $\mathrm{\mu g} / \mathrm{kg} / \mathrm{min}$ )-Protokolls kann Dobutamin aufgrund seiner positiven inotropen Wirkung auch zur Vitalitätsdiagnostik verwendet werden.

Im Rahmen der Stressuntersuchung zur Ischämiediagnostik wird eine Dobutamininfusionslösung über einen Perfusor (50 ml) in aufeinanderfolgenden Konzentrationen verabreicht, bis die Belastungszielherzfrequenz [0,85 x (220 - Alter) $]$ erreicht ist. Die Belastung wird von $10 \mu \mathrm{g} / \mathrm{kg} / \mathrm{min}$ bis $40 \mu \mathrm{g} / \mathrm{kg} /$ min ca. alle 3 min gesteigert bzw. dauert pro Belastungsstufe so lange, bis ein Bilddatensatz von CINE-MRT-Bildern in drei repräsentativen Kurzachsenschnitten, dem 4-Kammer-Blick und dem 2-Kammer-Blick, lange Achse mittels SSFP-Sequenzen 
aufgenommen worden ist. Bis zum Erreichen der vorher errechneten Zielherzfrequenz wird diese Bilddatenakquisition kontinuierlich wiederholt, bis neue Wandbewegungsstörungen auftreten bzw. andere Abbruchkriterien Anwendung finden. Sollte die Zielherzfrequenz ohne das Auftreten von neuen Wandbewegungsstörungen oder anderen Abbruchkriterien auf der höchsten Belastungsstufe noch nicht erreicht sein, kann noch Atropin i. v. appliziert werden.

\section{Überwachung des Patienten/Grundvoraussetzung für Belastungsuntersuchungen}

Wie bei allen anderen Belastungsuntersuchungen des Herzens müssen die Patienten während der Untersuchung sorgfältig von einem qualifizierten Arzt überwacht werden. Ein komplett ausgerüsteter Notfallkoffer mit Defibrillator und Notfallmedikation muss in unmittelbarer Nähe des MR-Scanners zur Verfügung stehen.

Da einige Notfallmaßnahmen wie Defibrillation und Intubation des Patienten im MRT nicht möglich sind, ist es vor allem notwendig, im Notfall den Patienten in kürzester Zeit aus dem MRT - ggf. unter fortlaufender Herzdruckmassage - bergen zu können. Hierzu eignen sich besonders abkoppelbare Tische bzw. ein Trolley, mit dem der MR-Tisch ohne Umlagerung des Patienten aus dem Scannerraum gefahren werden kann. Diesen Prozess sollte man mit dem MR-Team durchsprechen, trainieren und regelmäßig ebenso wie die Reanimation schulen.

\section{CHECKLISTE}

- nicht invasive Blutdruckmessung

- EKG-Überwachung

- Pulsoxymetrie

- Notfallklingel für den Patienten

- kontinuierliche Sprechverbindung

- visuelle Patientenüberwachung - Kamera

\section{Durchführung des Scans}

Bei Durchführung einer Stressherzuntersuchung beginnt man zunächst mit einem Localizer/Survey in koronarer, sagittaler und axialer Ausrichtung sowie ggf. einer axialen DarkBlood-TSE-Sequenz (z. B. HASTE) in Atem-Anhalte-Technik oder einer SSFP-„Whole-Heart-Sequenz“ in freier Atmung mittels Navigatortechnik. Hiermit könne alle kardiovaskulären und thorakalen Strukturen dargestellt werden.

Im Anschluss werden die kardialen Standardschnittebenen (2-Kammer-Blick, 4-Kammer-Blick) mittels CINE-SSFP-Sequenzen, an denen die Perfusionsmessung in drei repräsentativen Kurzachsenschnitten über den linken Ventrikel geplant wird, eingestellt. Die Perfusionsmessung wird üblicherweise zunächst als Test nativ und in Ruhe gemessen, um Einfaltungen noch vor Belastungsbeginn entdecken und vermeiden zu können. Dann erst erfolgt die Stressphase. Zur besseren Erfassung des Apex erlauben es einige Scanner, die drei repräsentativen Kurzachsenschnitte zusätzlich mit einem 4- oder 2-Kammer-Blick zur Perfusionsanalyse zu kombinieren. Im Gegensatz zur nuklearmedizinischen SPECT-Untersuchung werden nach wie vor in der Regel keine den linken Ventrikel komplett abdeckenden 3-D-Sequenzen eingesetzt. Diese sind zwar verfügbar und wurden auch schon in zahlreichen Studien eingesetzt, weisen aber meist noch eine verminderte zeitliche und räumliche Auflösung auf.

Vor Beginn der Belastungsuntersuchung und in regelmäßigen Abständen (z. B. jede Minute) während und nach der Belastung werden die Vitalparameter

- Herzfrequenz

- Blutdruck

überprüft und dokumentiert. Ein Aufenthalt eines Arztes im Scannerraum selbst während der Belastung ist nicht unbedingt erforderlich, wird aber u. a. zur Beruhigung des Patienten und zum schnellen Erfassen von Komplikationen und Einleiten von Notfallmaßnahmen empfohlen. Die Komplikationsrate bei Adenosin-Stress-MRT-Untersuchungen ist allerdings mit $0,12-0,17 \%$ sehr gering. Schwere Komplikationen sind äußerst selten.

Vor einer Adenosinbelastung kann der Patient evtl. noch einmal aus der Gantry herausgefahren werden, da die MRBilddatenakquisition erst nach Erreichen eines Gleichgewichts nach Gabe des Stressmedikaments (in der Regel nach 3 min) begonnen wird. Dann wird der Patient wieder in das Isozentrum des Scanners gefahren. Bei Verwendung von Adenosin wird dieses über einen MR-kompatiblen Injektor mit einer Dosierung von $140 \mu \mathrm{g} / \mathrm{kg} / \mathrm{min}$ appliziert. Dann beginnt die Perfusionsmessung, gleichzeitig wird Kontrastmittel über einen Injektor mit einer Geschwindigkeit von mindestens $3 \mathrm{ml} / \mathrm{s}$ verabreicht. Die Perfusionsmessung sollte in Atemstillstand über eine Dauer von mindestens 40-60 Herzschlägen erfolgen. Es empfiehlt sich deshalb ein zweigeteiltes Atemkommando:

- kurz vor Start der Messung

- kurz vor der Ankunft des Kontrastmittelbolus

durchzuführen. Ansonsten soll der Patient dazu angehalten werden, die Luft solange anzuhalten wie er kann und dann flach weiterzuatmen, bis der Scan beendet ist. Es empfiehlt sich, dies mit dem Patienten vorher zu üben. Wird Rapiscan als Stressor verwendet, erfolgt ein einmaliger Bolus von Hand mit einer Dosierung von 400 mg / 5 ml (! nicht gewichtsadaptiert!).

Zwischen der Perfusionsmessung in Stress und in Ruhe sollte man eine Wartezeit von 10-20 min einhalten. Diese Zeit kann genutzt werden, um Cine-Sequenzen in Kurzachsenausrichtung und - je nach Bedarf - in einer LVOT-Ebene zur Funktionsanalyse durchzuführen. 
Die sog. „Late-Enhancement“-Untersuchung erfolgt im Anschluss an die Perfusionsmessung in der Ruhephase. Eine Ruheperfusionsmessung ist nicht unbedingt erforderlich, da es anders als in der Nuklearmedizin beim Thallium oder Technetium nicht zu einer „Redistribution“ von Speicherdefekten kommt. Ein nicht mehr Vorhandensein von Perfusionsdefiziten in der Ruhephase kann somit nicht als Vitalität des Herzmuskels gedeutet werden wie in der Myokardszintigrafie. Zur Vitalitätsdiagnostik dient die anschließende „Late-Enhancement“-Aufnahme in der MRT. Wird jedoch bei der Ruheperfusion nur 0,05 mmol/ kg Körpergewicht Gd-DTPA appliziert, ist eine zweite Kontrastmittelgabe zum Erreichen von mindestens 0,1-0,2 mmol / kg Körpergewicht Gd-DTPA Gesamtkontrastmittelmenge für das „Late Gadolinium Enhancement" (LGE) erforderlich.

Technik der verzögerten Kontrastmittelanreicherung zur Vitalitäts- bzw. Narbendiagnostik

Das Prinzip der verzögerten Kontrastmittelanreicherung „Late Enhancement“ beruht - vereinfacht erklärt - sowohl auf einer verzögerten Anreicherung als auch einem verzögerten „Auswaschen“ von gadoliniumhaltigem MR-Kontrastmittel in Myokardnekrosen oder Narben. Das Kontrastmittel reichert sich in nekrotischem Gewebe sowohl intra- als auch extrazellulär an. Im akuten Stadium erfolgt das durch eine ödembedingte Vergrößerung des Extrazellularraums, im chronischen Stadium durch den Ersatz des untergegangenen Myokards durch Bindegewebe, also der Ausbildung einer Myokardnarbe. Durch eine spezielle Form von Inversionspulsen kann das gesunde Myokard „genullt“, also ohne Signal und damit „schwarz" dargestellt werden, während sich das Narbengewebe hell darstellt („Bright is dead“). Typischerweise wird eine einfachedoppelte Dosis Kontrastmittel (0,1-0,2 mmol / kg Körpergewicht) appliziert und 10-20 min nach Kontrastmittelgabe mithilfe von Inversion-RecoveryGradientenecho-Sequenzen (IR-GRE) Aufnahmen angefertigt. Um das gesunde Myokard zu „nullen“, bedarf es der geeigneten Wahl der Inversionszeit (TI). Hierzu stehen spezielle schnelle Sequenzen (z. B. LookLocker oder TI-Scout), mit dessen Hilfe die beste TI-Zeit bestimmt werden kann, zur Verfügung. Üblicherweise liegt die optimale Inversionszeit zwischen 200-300 ms ( $\triangleright$ Abb. 10 und $\triangleright$ Abb. 11).

\footnotetext{
Merke

Da Perfusionsdefekte unter pharmakologischer Belastung nicht nur durch eine myokardiale Ischämie, sondern auch durch abgelaufene Myokardinfarkte hervorgerufen werden können, ist die Stressperfusion immer mit einer LGEUntersuchung zu kombinieren.
}

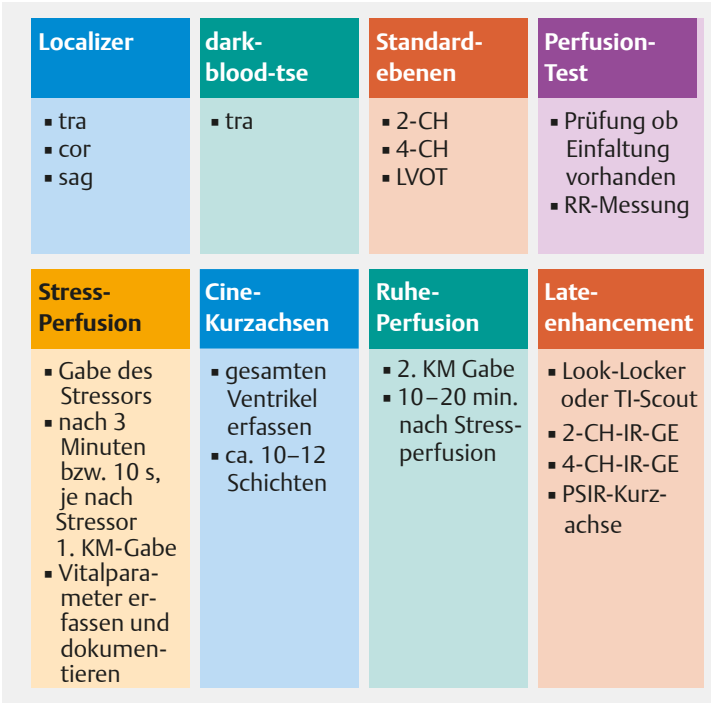

Abb. 10 Schematische Darstellung eines Stressprotokolls für die Adenosin-/Regadenoson-/Dipyridamolbelastung.

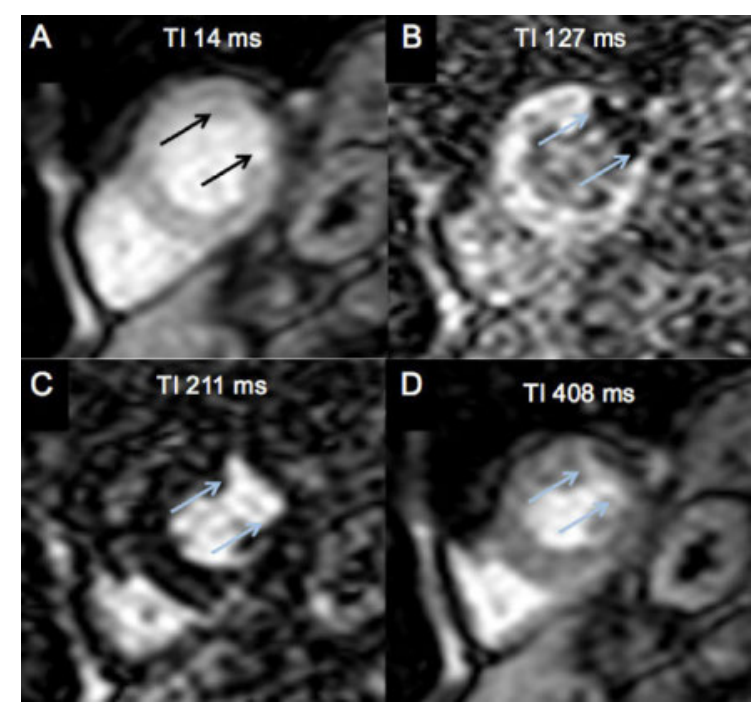

- Abb. 11 Narbendarstellung mit Look-Locker-Sequenz. Exemplarisch ausgewählte Bilder einer dynamischen LookLocker-Sequenzserie nach Kontrastmittelgabe eines Patienten mit chronischem Infarkt im Bereich der Lateralwand des linken Ventrikels (a-d, Pfeile) unter Verwendung unterschiedlicher Inversionszeiten (Bildquelle: Gutberlet M. Bildgebende Diagnostik angeborener Herzfehler. Stuttgart: Thieme; 2016).

a Bei einer Inversionszeit von 14 ms zeigen sich sowohl im vitalen Myokard als auch in der Narbe annähernd gleiche Signalintensitätswerte.

b Bei einer Inversionszeit von 127 ms hat die Narbe ihren Nulldurchgang und wird schwarz dargestellt (Pfeile).

c Optimale Wahl der Inversionszeit mit 211 ms: Das gesunde Myokard hat seinen Nulldurchgang und wird schwarz, die Narbe hell dargestellt.

d Bei Wahl einer zu langen Inversionszeit (hier 408 ms) sind Narbe und vitales Myokard erneut nicht zu differenzieren. 
Mittlerweile gibt es auch Sequenzen, die weniger anfällig sind für eine korrekte Wahl der Inversionszeit, sog. „Phasen-Sensitive Inversion Recovery“ (PSIR)-Sequenzen, die jeweils aus mehreren Bildern bestehen. In der Regel wird nur das Phasenbild zur Diagnostik herangezogen.

\section{Auswertung}

Die Auswertung der Messung schließt sich an die Messung und ggf. an den Transfer der Bilddaten auf einen speziellen Auswerterechner des MRT-Systems an.

Nach der Bolusinjektion wird das Kontrastmittel durch das Blut ins Myokard transportiert. Es kommt zunächst zu einer Einwaschphase von subepikardial nach endokardial, die unter den meisten Injektionsbedingungen subendokardial dann nahtlos in eine zeitlich relativ konstante Signalintensität übergeht. Im Falle einer Ischämie flutet das Kontrastmittel wesentlich langsamer an. Innerhalb einer Atemanhalteperiode wird kein Signalmaximum erreicht, die Signalintensität bleibt unterhalb der Signalintensität des normal perfundierten Myokards. Das heißt, eine Ischämie zeigt sich relativ zu normal perfundierten Myokard negativ kontrastiert: je ausgeprägter die Ischämie desto deutlicher der Kontrast. Physiologischerweise beginnt das Perfusionsdefizit ( $\triangleright$ Abb. 12) subendokardial und breitet sich flächig nach subepikardial aus.

Im Extremfall, beispielsweise bei einem Verschluss einer Koronararterie ohne Kollateralversorgung, flutet kein Kontrastmittel an, d. h. es findet keine Perfusion statt.

Für viele klinische Fragestellungen genügt eine qualitative Bildanalyse, die mit geringem Aufwand durchgeführt werden kann. Bei erhöhten Anforderungen an die Genau- igkeit und Vergleichbarkeit der Messungen ist eine semiquantitative Analyse der Bilddaten empfehlenswert. Eine quantitative Analyse ist derzeit nur bei wissenschaftlichen Fragestellungen sinnvoll.

\section{Merke}

Für die semiquantitative und für die quantitative Analyse von Perfusionsmessungen ist eine gute EKG-Triggerung sowie eine stabile Atem-AnhaltePhase erforderlich. Sind diesen Bedingungen nicht erfüllt, kann es leicht zu großen Messfehlern kommen.

\section{KERNAUSSAGEN}

- Ein Kardio-CT bzw. eine Kardio-MRT ist optimal für alle Patienten mit „mittlerer“ Vortestwahrscheinlichkeit auf eine koronare Herzerkrankung (KHK) geeignet.

- Eine Kardio-CT wird zur Beurteilung der Koronarmorphologie benutzt.

- Mithilfe der Kardio-MRT kann die Myokarddurchblutung und Vitalität beurteilt werden.

- Die Komplikationsrate einer Adenosin-/Regadenoson-Stress-MRT ist mit $<0,2 \%$ sehr gering.

- Eine Herzfrequenzsenkung und Nitroglyceringabe sind für eine gute Bildqualität bei der Koronar-CT essenziell wichtig.

- Zu einer Stress-MRT „First-Pass“-Perfusionsanalyse gehört immer auch eine Vitalitätsbeurteilung mittels „Late-GadoliniumEnhancement“ (LGE).
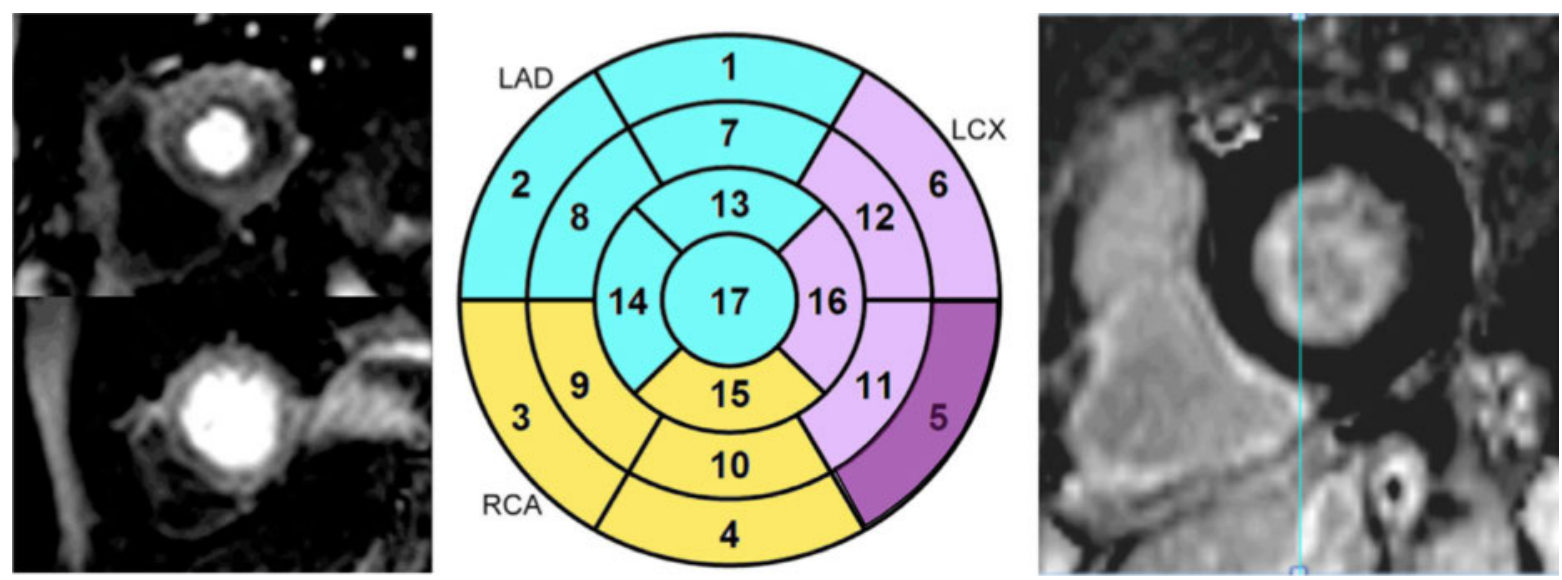

Abb. 12 Stress-MRT-Untersuchung mit Adenosinbelastung ( $140 \mu \mathrm{g} / \mathrm{kg} / \mathrm{min}$ über 3 min) einer 55-jährigen Patientin mit EingefäßKHK der LCX. Links: eindeutiges flächiges Perfusionsdefizit im Bereich der Seitenwand des linken Ventrikels - Versorgungsgebiet der linken Circumflexarterie (LCX) in Segment 5 des 17-Segment-Modells nach AHA (Mitte). Kein Nachweis einer myokardialen Narbe im LGE-Bild (rechts). 


\section{Interessenskonflikt}

Die Autoren geben an, dass kein Interessenkonflikt besteht.

\section{Autorinnen/Autoren}

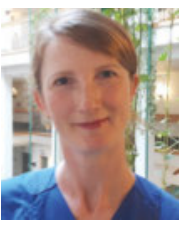

\section{Susan Rosemeier}

Jahrgang 1984. 2003-2006 Ausbildung zur med-.techn. Radiologieassistentin an der Otto von Guericke Universität in Magdeburg. 2006-2008 Tätigkeit als MTRA am St. Bernward Krankenhaus. Seit 2008 als MTRA an dem Herzzentrum Leipzig tätig. Seit

Dezember 2015 leitende MTRA der Radiologischen Abteilung am Herzzentrum Leipzig und Mentorin für Auszubildende der medizinischen Fachschule des Universitätsklinikum in Leipzig.

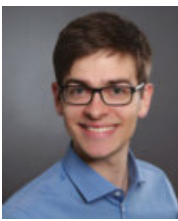

\section{Christopher Berger}

Jahrgang 1992. 2013-2016 Ausbildung zum MTRA am Universitätsklinikum Hale (Saale).

Seit 2016 MTRA im Herzzentrum Leipzig Radiologie.

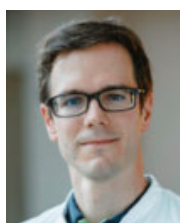

Christian Krieghoff, Dr. med.

Jahrgang 1981. 2002-2008 Medizinstudium in Magdeburg und Leipzig. 2009-2016

Assistenzarzt in der Abteilung für Diagnostische und Interventionelle Radiologie am Herzzentrum Leipzig. 2016 Facharztprüfung Radiologie und Promotion über Herz-MRT zur Abstoßungsdiagnostik nach Herztransplantation. Seit 2016 Oberarzt am Herzzentrum Leipzig und CT-Geräteverantwortlicher (Somatom Definition Flash).

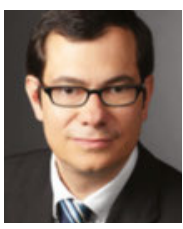

\section{Matthias Gutberlet, Prof. Dr. med, EBCR} 1985-1992 Studium der Humanmedizin, Philipps-Universität Marburg. 1992-2000 Assistenzarzt Deutsches Herzzentrum Berlin und Klinik für Strahlenheilkunde, Charité, Campus Virchow-Klinikum; bis 2007 wissenschaftlicher Assistent Klinik für Strahlenheilkunde und seit 2007 Chefarzt der Abt. für Diagnostische und Interventionelle Radiologie, Herzzentrum Leipzig, W2-Stiftungsprofessur für „kardiologische Bildgebung in der Radiologie“ Universität Leipzig.

\section{Korrespondenzadresse}

\section{Prof. Dr. med. Matthias Gutberlet}

Herzzentrum Leipzig

Abt. für Radiologie

Strümpellstr. 39

04289 Leipzig

Deutschland

Tel: $+49(0) 341 / 865-0$

Fax: $+49(0) 341 / 865-1405$

Matthias.Gutberlet@helios-kliniken.de

\section{Literaturverzeichnis}

[1] Fischbach R, Keithahn A. Koronare Herzkrankheit. Radiopraxis 2009; 2: 197-206. doi:10.1055/s-0029-1244939

[2] Gutberlet M. Kardiale Magnetresonaztomographie. Vom Bild zur Diagnose. Radiologe. 2013; 53: 1033-1052. PMID: 24231826
[3] Achenbach S, Barkhausen J, Beer M et al. Consensus recommodendations of the German Radiology Society (DRG), the German Cardiac Society (DGK) and the German Society for Pediatric Cardiology (DGPK) on the use of cardiac imaging with computed tomografy and magnetic resonance imaging. Rofo 2012, 184: 345-368

[4] Montalescot G, Sechtem U, Achenbach S et al. 2013 ESC guidelines on the management of stable coronary artery disease: the Task Force on the management of stable coronary artery disease of the European Society of Cardiology. Eur Heart J. 2013; 34: 2949-3003. Erratum in: Eur Heart J. 2014; 35: 2260-2261. PMID: 23996286

[5] Bundesärztekammer (BÄK), Kassenärztliche Bundesvereinigung (KBV), Arbeitsgemeinschaft der Wissenschaftlichen Medizinischen Fachgesellschaften (AWMF): Nationale VersorgungsLeitlinie Chronische KHK - Langfassung, 4. Aufl. Version 1/2016. Im Internet: www.khk.versorgungsleitlinien. de; Stand: 12.09.2017. doi:10.6101/AZQ/000267

[6] Im Internet: www.versorgungsleitlinien.de, Stand: 24.10.2017

[7] Im Internet: www.awmf-leitlinien.de; Stand: 24.10.2017

[8] Schuhbäck A, Kolwelter J, Achenbach S et al. DiamondForrester and cardiac CT: Is there a need to redefine the pretest probability of coronary artery disease? Herz 2016; 41: 371-375. doi:10.1007/s00059-016-4437-1

[9] Athanasiadis A, Sechtem U. Diagnostik und Therapie der chronisch stabilen koronaren Herzkrankheit - Neue Empfehlungen der Europäischen Gesellschaft für Kardiologie. Herz 2014, 39: 902-912

[10] Kardiales MR/CT-Registry der European Society of Cardiovascular Radiology (ESCR). Im Internet: www.mrct-registry.org; Stand: 24.10.2017

[11] Becker C, Fuchs E. Herzbildgebung: Szintigrafie versus Computertomografie - Konkurrenz oder Ergänzung? Radiopraxis 2013; 6: 71-87. doi:10.1055/s-0033-1344032

[12] Abbara S, Blanke P, Maroules CD et al. SCCT guidelines for the performance and acquisition of coronary computed tomographic angiography: A report of the society of Cardiovascular Computed Tomography Guidelines Committee Endorsed by the North American Society for Cardiovascular Imaging (NASCl). J Cardiovasc Comput Tomogr 2016; 10: 435-449

[13] Foley S], McEntee MF, Achenbach S et al. Breast Surface Radiation Dose During Coronary CT Angiography: Reduction by Breast Displacement and Lead Shielding. Am J Radiol 2011; 197: 367-373

[14] Alkadhi H, Leschka S, Stolzmann P, Flohr Th. Praxisbuch Herz-CT. 2. Aufl. Heidelberg: Springer; 2013

[15] Small GR, Kazmi M, Dekemp RA et al. Established and emerging dose reduction methods in cardiac computed tomography. J Nucl Cardiol 2011; 18: 570-579

[16] Bundesamt für Strahlenschutz. Bekanntmachung der aktualisierten diagnostischen Referenzwerte für diagnostische und interventionelle Röntgenanwendungen. Bundesanzeiger 2016; B8

[17] Menke J, Kowalski J. Diagnostic accuracy and utility of coronary $\mathrm{CT}$ angiography with consideration of unevaluable results: A systematic review and multivariate Bayesian random-effects meta-analysis with intention to diagnose. Eur Radiol 2016; 26: 451-458

[18] Koo H], Yang DH, Kim YH. CT-based myocardial ischemia evaluation: quantitative angiography, transluminal attenuation gradient, myocardial perfusion, and CT-derived fractional flow reserve. Int ] Cardiovasc Imaging 2016; 32 (Suppl. 1): S1-S19 


\section{Punkte sammeln auf CME.thiemede}

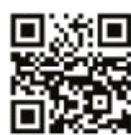

Diese Fortbildungseinheit ist 12 Monate online für die Teilnahme verfügbar.

Sollten Sie Fragen zur Online-Teilnahme haben, finden Sie unter http://cme.thieme.de/hilfe eine ausführliche Anleitung. Wir wünschen viel Erfolg beim Beantworten der Fragen!

Unter eref.thieme.de/ZZX8MFK oder über den QR-Code kommen Sie direkt zum Artikel zur Eingabe der Antworten.

\section{CPD-Fragen}

\section{Frage 1}

Für welche Patienten mit Verdacht auf das Vorliegen einer KHK ist das Stress-MRT bzw. eine Koronar-CT entsprechend der aktuellen Leitlinien und der aktuellen Versorgungsleitlinie chronische KHK geeignet?

A Für Patienten mit einer sehr hohen Vortestwahrscheinlichkeit für das Vorliegen einer KHK (> $90 \%$ ) sind Kardio-CT und Kardio-MRT besonders geeignet.

B Für Patienten mit einer sehr niedrigen Vortestwahrscheinlichkeit für das Vorliegen einer KHK (<10\%) sind Kardio-CT und Kardio-MRT besonders geeignet.

C Alle Patienten mit Verdacht auf das Vorliegen einer KHK sollten eine invasive Herzkatheteruntersuchung erhalten.

D Für Patienten mit einer „intermediären“ bzw. mittleren Vortestwahrscheinlichkeit für das Vorliegen einer KHK (zwischen 15-85\%) sind Kardio-CT und Kardio-MRT besonders geeignet.

E Alle Patienten mit Verdacht auf das Vorliegen einer KHK sollten eine Kardio-MRT oder Kardio-CT Untersuchung erhalten.

\section{Frage 2}

Welche 3 Faktoren werden für die Abschätzung der Vortestwahrscheinlichkeit auf das Vorliegen einer KHK nach dem Diamond-Forrester Score genutzt?

A Körpergröße, Körpergewicht und der Body-Mass-Index (BMI)

B Patientenalter, Patientengeschlecht, Patientenbeschwerdesymptomatik

C Dauer des Nikotinkonsums, Höhe der Blutfette, Höhe des Blutdrucks

D Blutzuckerspiegel, Glomeruläre Filtrationsrate (GFR), Höhe des Blutdrucks

E Auftreten von Herzinfarkten, Schlaganfällen und peripheren Gefäßverengungen bei Verwandten 1. Grades

\section{Frage 3}

Welche Medikamente sollten für die Durchführung einer Koronar-CT zur Verbesserung der Bildqualität immer zur Verfügung stehen und eingesetzt werden, falls keine Kontraindikationen bestehen?

A Kalziumantagonisten und ACE-Hemmer

B Digitoxin und Digoxin

C Adenosin und Regadenoson
D Betablocker und Nitrospray

E Dopamin und Dobutamin

\section{Frage 4}

Welches sind die optimalen Bedingungen bezüglich Herzfrequenz und Herzrhythmus für eine Koronar-CT?

A eine hohe Herzfrequenz > 100 / min und Vorhofflimmern

$B$ eine niedrige Herzfrequenz $<60-70$ / min und regelmäßiger Sinusrhythmus

C eine mittlere Herzfrequenz von 80-100 / min und unregelmäBiger Sinusrhythmus

D eine niedrige Herzfrequenz von 50 / min und Arrhythmien

E eine hohe Herzfrequenz und regelmäßiger Sinusrhythmus

\section{Frage 5}

Wie soll der Patient bei einer Koronar-CT gelagert werden?

A in Bauchlage, Körper im Isozentrum, Arme neben dem Körper

$B$ in Rückenlage, Körper im Isozentrum, Arme oben hinter dem Kopf

C in Bauchlage, Herz im Isozentrum, Arme neben dem Körper

D in Rückenlage, Herz im Isozentrum, Arme oben hinter dem Kopf

E In Bauchlage, Herz im Isozentrum, Arme oben hinter dem Kopf

\section{Frage 6}

Welche Aussage zur optimalen Herzphase für die Koronararterienrekonstruktion bei der Koronar-CT ist richtig?

A Bei Verwendung von retrospektivem Gating sollte unabhängig von der Herzfrequenz immer in der Systole (Herzaustreibungsphase) rekonstruiert werden.

B Bei Verwendung des prospektiven Gatings mittels „Step and Shoot" sollte unabhängig von der Herzfrequenz immer in der Systole (Herzaustreibungsphase) rekonstruiert werden.

C Bei Verwendung des retrospektiven Gating sollte bei niedriger Herzfrequenz (<60-70 / min) in der mittleren Diastole (Herzfüllungsphase) bei 55-65 \% des RR-Intervalls rekonstruiert werden.

D Bei Verwendung des prospektiven Gatings mittels „Step and Shoot" sollte bei hoher Herzfrequenz immer in der frühen Systole (Herzaustreibungsphase) bei 0-20\% des RR-Intervalls rekonstruiert werden.

E Bei Verwendung von retrospektivem Gating sollte unabhängig von der Herzfrequenz immer in der Diastole (Herzfüllungsphase) rekonstruiert werden. 


\section{CPD-Fragen}

Fortsetzung $\ldots$

\section{Frage 7}

Welche Nahrungsmittel sollte der Patient mindestens 6-12 h, besser 24 h vor einer Adenosin-/RegadenosonStress-MRT-Untersuchung nicht zu sich genommen haben?

A Schweine-, Rind- und Lammfleisch

B Milch-, Ei- und Sojaprodukte

C Grünen Salat, Spinat und Brokkoli

D Eiweißhaltige Nahrungsmittel

E Kaffee, Tee und Schokolade

\section{Frage 8}

Welche Aussagen zum Wirkmechanismus von Adenosin und Dobutamin bei der Stress-MRT sind richtig?

A Adenosin und Regadenoson wirken direkt herzfrequenzsteigernd über eine positiv chronotrope Wirkung am ß1-Rezeptor.

B Adenosin und Regadenoson wirken direkt gefäßerweiternd über den Adenosinrezeptor.

C Dobutamin wirkt direkt gefäßerweiternd über den ß1-Rezeptor.

D Dobutamin führt in relevant eingeengten Koronargefäßen über einen sog. „Steal Effect“ zu einer myokardialen Minderdurchblutung.

E Adenosin/Regadenoson und Dobutamin haben den gleichen Wirkmechanismus.

\section{Frage 9}

Welche Aussagen zur verzögerten Kontrastmittelanreicherung („Late Gadolinium Enhancement“ - LGE) sind richtig?

A Die typische zu wählende Inversionszeit ( $\mathrm{TI}$ ) bei Verwendung einer IR-GRE-Sequenz für das LGE 10-20 min nach Kontrastmittelgabe beträgt 200$300 \mathrm{~ms}$.
B Die typische zu wählende Inversionszeit ( $\mathrm{TI}$ ) bei Verwendung einer IR-GRE-Sequenz für das LGE 10-20 min nach Kontrastmittelgabe beträgt 20-30 ms.

C Die typische zu wählende Inversionszeit ( $\mathrm{TI}$ ) bei Verwendung einer PSIR-Sequenz für das LGE 10-20 min nach Kontrastmittelgabe beträgt 2000-3000 ms.

D Die typische zu wählende Inversionszeit bei Verwendung einer PSIR-Sequenz für das LGE 10-20 min nach Kontrastmittelgabe beträgt 2-3 ms.

E Eine korrekte Wahl der Inversionszeit (TI) ist bei Verwendung einer IR-GRE-Sequenz für das LGE 10-20 min nach Kontrastmittelgabe nicht notwendig.

\section{Frage 10}

Welche Aussagen zur „First-Pass“-MR-Perfusionsanalyse bei der Stress-MRT sind nicht richtig?

A Vor Kontrastmittelgabe und Beginn der Adenosinbelastung sollte ein Testscan der Perfusionssequenz laufen.

B Die „First-Pass“-MR-Perfusionsanalyse bei der Stress-MRT mit Adenosin/Regadenoson erfolgt in der Regel rein visuell.

C Das Adenosin wird üblicherweise in einer gewichtsangepassten Dosierung von 140 mg / kg Körpergewicht/ min i. v. über 6 min eingesetzt.

D Der selektive Adenosinrezeptoragonist Regadenoson wird ohne Gewichtsadaptation als Bolus ( $5 \mathrm{ml}$ ) über $10 \mathrm{~s}$ in einer Dosierung von $400 \mu \mathrm{g}$ i. v. appliziert

E Die Komplikationsrate einer Adenosin-/RegadenosonStress-MRT ist sehr hoch und liegt bei bis zu $5 \%$. 